\title{
Multiple Criteria Hierarchy Process for ELECTRE Tri methods
}

\author{
Salvatore Corrente ${ }^{\mathrm{a}}$, Salvatore Greco ${ }^{\mathrm{a}, \mathrm{d}}$, Roman Słowiński $^{\mathrm{b}, \mathrm{c}}$ \\ ${ }^{a}$ Department of Economics and Business, University of Catania, Corso Italia, 55, 95129 Catania, Italy \\ ${ }^{b}$ Institute of Computing Science, Poznań University of Technology, 60-965 Poznań, Poland \\ ${ }^{c}$ Systems Research Institute, Polish Academy of Sciences, 01-447 Warsaw, Poland \\ ${ }^{d}$ University of Portsmouth, Portsmouth Business School, Centre of Operations Research and Logistics (CORL), Richmond \\ Building, Portland Street, Portsmouth PO1 3DE, United Kingdom
}

\begin{abstract}
Sorting methods, in particular ELECTRE Tri methods, are widely used in Multiple Criteria Decision Aiding to deal with ordinal classification problems. Problems of this kind encountered in practice involve the evaluation of different alternatives (actions) on several evaluation criteria that are structured in a hierarchical way. In order to deal with a hierarchical structure of criteria in decision problems, Multiple Criteria Hierarchy Process (MCHP) has been recently proposed. In this paper, we apply the MCHP to the ELECTRE-Tri methods. In particular, we extend ELECTRE Tri-B, ELECTRE Tri-C and ELECTRE Tri-nC methods. We also adapt the MCHP concept to the case where interaction among evaluation criteria has either strengthening, or weakening, or antagonistic effect. Finally, we present an extension of the SRF method to determine the weights of criteria in case they are hierarchically structured.
\end{abstract}

Keywords: Multiple criteria decision aiding, Sorting problems, Multiple Criteria Hierarchy Process, ELECTRE Tri

\section{Introduction}

Multiple Criteria Decision Aiding (MCDA) methods deal with three major types of problems: choice, ranking and sorting (for a collection of surveys on MCDA see [12]). Given a finite set of alternatives, choice problems consist in choosing a subset of best alternatives among the considered ones; ranking problems consist in rank ordering all the considered alternatives from the best to the worst, while sorting problems consist in assigning each alternative to one of the predefined and ordered categories (decision classes).

Among the best known sorting methods, there are the ELECTRE Tri methods and, in particular, the ELECTRE Tri-B [21], the ELECTRE Tri-C [2] and the ELECTRE Tri-nC [3] methods (for other sorting methods see $[17,18])$.

Email addresses: salvatore.corrente@unict.it (Salvatore Corrente), salgreco@unict.it (Salvatore Greco), roman.slowinski@cs.put.poznan.pl (Roman Słowiński)

[Post-print] Please cite as: Corrente Salvatore, Greco Salvatore, Słowiński Roman. Multiple Criteria Hierarchy Process for ELECTRE Tri methods. European Journal of Operational Research, 252(1), 191-203 
Given a set of alternatives $A=\left\{a_{1}, \ldots, a_{n}\right\}$ evaluated with respect to a set of evaluation criteria $\mathcal{G}$, and $p$ categories $C l_{1}, \ldots, C l_{p}$ ordered from the worst to the best (such that $C l_{h+1} \succ C l_{h}$, for all $h=1, \ldots, p-1$ ), the ELECTRE Tri methods aim at assigning each alternative of $A$ to one or more contiguous categories. In ELECTRE Tri-B, each category $C l_{h}$ is delimited by two reference profiles $b_{h-1}$ and $b_{h}$. The reference profiles $b_{h-1}$ and $b_{h}$ are like fictitious alternatives evaluated on all criteria from set $\mathcal{G}$. They are limiting category $C_{h}$ from the bottom and the top, respectively. In ELECTRE Tri-C and ELECTRE Tri-nC methods, each category is characterized by one reference profile or more than one reference profile, respectively which correspond to some typical objects of this category. In all three methods, the assignment of alternatives to categories depends on the comparison of these alternatives with the reference profiles corresponding to the categories, the comparison that takes into account the whole set of criteria. It is worth mentioning that the assignment of one alternative is not influenced by the assignment of another alternative. Recently, Bouyssou and Marchant investigated in [6] the relationship between ELECTRE Tri-B and ELECTRE TriC, providing also another variant of ELECTRE Tri-B. On one hand, they demonstrated that some sorting assignments obtained by ELECTRE Tri-B cannot be obtained by fixing adequate profiles in ELECTRE Tri-C and viceversa; on the other hand, the authors adapted ELECTRE Tri-B so that the pessimistic and the optimistic recommendations can be obtained one from the other by a transposition operation ${ }^{2}$.

In all known sorting methods, all evaluation criteria are considered at the same level. However, practical decision problems often require consideration of a hierarchical structure of the set of criteria. Considering a hierarchy of criteria is also a way of decomposing complex decision problems involving criteria referring to various levels of detail in the evaluation. For example, let us consider a council that evaluates a set of projects with respect to different aspects: economic, environmental, social, etc. In order to make these evaluations, the council has to consider several indicators for each macro criterion, and each indicator is composed, in turn, of other subcriteria, and so on. Even if global evaluation of a single project is well appreciated, members of the council would appreciate having an insight into partial evaluation of the projects, referring to a greater level of detail, e.g., with respect to economic, environmental or social aspects considered separately. The Multiple Criteria Hierarchy Process (MCHP) has been recently proposed for this purpose. It deals with decision problems where criteria are structured hierarchically, and at each level of the hierarchy, they are aggregated using one of available aggregation methods: either those involving additive [10] or non-additive [4] value functions, or those involving outranking relations [11].

The three main novelties introduced in this paper are:

\footnotetext{
${ }^{2}$ According to [6], the transposition operation consists in inverting the direction of preference on all criteria and in inverting the ordering of the categories.
} 
- MCHP has been applied to ELECTRE Tri-B, ELECTRE Tri-C and ELECTRE Tri-nC in a way which permits to sort the alternatives into different categories not only in the root, at the comprehensive level, but also with respect to a lower level criterion, represented by an intermediate node of the hierarchy tree. In the ELECTRE framework, we take into account three different outranking relations based on the concordance and non-discordance tests; we specify two coherence properties that should hold for outranking methods applied to problems involving criteria organized in a hierarchical way, and we provide necessary and sufficient conditions on the cutting of the credibility of outranking, such that these two properties are satisfied. Moreover, some theorems show the relationship between the assignments done by ELECTRE Tri-B at different levels of the hierarchy, using both the pessimistic and the optimistic assignment procedures. We show that in case of a flat structure of criteria, the proposed methods boil down to the classical ELECTRE Tri methods and, for this reason, they can be considered their generalizations;

- The Simos-Roy-Figueira (SRF) method [14], used to determine the weights of criteria in the classical ELECTRE methods, has been extended to handle criteria organized in a hierarchical way. In this case, following a top-down procedure, the weights of all criteria in the hierarchy, starting from the root criterion to the elementary criteria, are obtained. Even if the extension of the SRF method has been proposed in this paper to deal with sorting problems, it can be applied to choice and ranking problems involving criteria organized in a hierarchical way if these problems are solved using methods in which weights are interpreted as in ELECTRE methods, for example, when using PROMETHEE methods [7];

- A methodology for dealing with different types of interactions between criteria organized in a hierarchical way has been proposed; extending the proposal of [13], in this paper we explain how to deal with decision making problems in which criteria organized in a hierarchy can present different types of interactions, such as synergy, redundancy, and antagonistic effects. Also in this case, even if this methodology has been proposed for dealing with sorting problems, it can be applied to choice and ranking problems approached by methods in which importance of criteria is interpreted as in ELECTRE methods.

The paper is organized as follows: Section 2 recalls the basic concepts of MCHP and ELECTRE Tri methods; in Section 3, we specify some coherence properties that should hold for hierarchical multiple criteria ELECTRE methods, as well as theorems ensuring them; in Section 4, we describe the application of MCHP to ELECTRE Tri methods, as well as their extension to the case of interacting criteria; an example of application of the proposed methodology to a real world decision making problem is presented in Section 
5, while some concluding remarks and further directions of research are pointed out in Section 6 .

\section{MCHP and a brief reminder of ELECTRE Tri methods}

In MCHP [10], we consider a set of criteria structured in a hierarchical way, i.e., criteria are not considered at the same level, but they are distributed over $l$ different levels; $\mathcal{G}$ denotes the entire set of criteria considered at all levels; $\mathcal{I}_{\mathcal{G}}$ is the set of indices of particular criteria representing positions of criteria in the hierarchy; $G_{\mathbf{r}}$ is a generic symbol of criterion from any level of the hierarchy; $n(\mathbf{r})$ is the number of subcriteria of $G_{\mathbf{r}}$ in the subsequent level, i.e., the direct subcriteria of $G_{\mathbf{r}}$ are $G_{(\mathbf{r}, 1)}, \ldots, G_{(\mathbf{r}, n(\mathbf{r}))} ; g_{\mathbf{t}}: A \rightarrow \mathbb{R}$ denotes an elementary criterion, i.e., a criterion at level $l$ of the hierarchy tree; $E L$ is the set of indices of all elementary criteria; $E\left(G_{\mathbf{r}}\right)$ is the set of indices of elementary criteria descending from $G_{\mathbf{r}} ; L B O$ is the set of indices of all subcriteria located at the last but one level of the hierarchy and $L B\left(G_{\mathbf{r}}\right)$ is the set of indices of the subcriteria descending from $G_{\mathbf{r}}$ located at the last but one level of the hierarchy (for a more detailed description of the notation used in MCHP, see [10]).

Let us mention that, in the following, we shall suppose without loss of generality that all elementary criteria have an increasing direction of preference (the greater the evaluation of an alternative on an elementary criterion, the better the alternative is), and that when $\mathbf{r}=0$, then by $G_{\mathbf{r}}=G_{\mathbf{0}}$ we mean the entire set of criteria and not a particular criterion or subcriterion; in this particular case, $E\left(G_{0}\right)=E L$ and $L B\left(G_{\mathbf{0}}\right)=L B O$.

For each elementary criterion $g_{\mathbf{t}}, \mathbf{t} \in E L$, the real number $w_{\mathbf{t}}$ represents a relative importance (weight) of $g_{\mathbf{t}}$ within the family of all elementary criteria, and we suppose, without loss of generality, that $\sum_{\mathbf{t} \in E L} w_{\mathbf{t}}=1$. The indifference, preference and veto thresholds for each elementary criterion $g_{\mathbf{t}}$ are denoted by $q_{\mathbf{t}}, p_{\mathbf{t}}$, and $v_{\mathbf{t}}$, respectively. $q_{\mathbf{t}}$ is the greatest difference between evaluations of alternative $a$ and $b$ on elementary criterion $g_{\mathbf{t}}$ compatible with the indifference among them; $p_{\mathbf{t}}$ is the smallest difference between evaluations of $a$ and $b$ on $g_{\mathbf{t}}$, being compatible with the preference of one alternative over the other; $v_{\mathbf{t}}$ is an upper bound beyond which the discordance about the outranking of one alternative over the other cannot surpass. For consistency, $0 \leq q_{\mathbf{t}} \leq p_{\mathbf{t}}<v_{\mathbf{t}}$. In the following, for the sake of simplicity, we shall consider constant thresholds only, however, this assumption can easily be relaxed.

Note 2.1. Let us stress that, as we shall explain in section 4, in ELECTRE Tri methods the assignment of an alternative to one of the considered categories depends on the comparison of the alternative with the reference profiles separating the categories. As the reference profiles can be seen as fictitious alternatives, the indices that we shall define in the following lines, are valid also for the the comparison between two alternatives. For this reason, in defining these indices, by $a$ and $b$ we mean two alternatives or, an alternative and a reference profile.

In all considered adaptations of ELECTRE Tri methods to MCHP, we shall define the following indices: 
- The elementary concordance index $\phi_{\mathbf{t}}(a, b)$ for each elementary criterion $g_{\mathbf{t}}$ :

$$
\phi_{\mathbf{t}}(a, b)= \begin{cases}1 & \text { if } \quad g_{\mathbf{t}}(b)-g_{\mathbf{t}}(a) \leq q_{\mathbf{t}},\left(a S_{\mathbf{t}} b\right), \\ \frac{p_{\mathbf{t}}-\left[g_{\mathbf{t}}(b)-g_{\mathbf{t}}(a)\right]}{p_{\mathbf{t}}-q_{\mathbf{t}}} & \text { if } \quad q_{\mathbf{t}}<g_{\mathbf{t}}(b)-g_{\mathbf{t}}(a)<p_{\mathbf{t}},\left(b Q_{\mathbf{t}} a\right), \\ 0 & \text { if } g_{\mathbf{t}}(b)-g_{\mathbf{t}}(a) \geq p_{\mathbf{t}},\left(b P_{\mathbf{t}} a\right) .\end{cases}
$$

It represents the degree of concordance with the hypothesis of outranking of $a$ over $b$ on the elementary criterion $g_{\mathbf{t}}$. It is a non-increasing function of the difference $g_{\mathbf{t}}(b)-g_{\mathbf{t}}(a)$. The binary relations assigned to the three above cases have the following meaning: $S_{\mathbf{t}}$ - outranking, $Q_{\mathbf{t}}$ - weak preference, $P_{\mathbf{t}}$ - strict preference, all of them with respect to $g_{\mathbf{t}}$.

- The elementary discordance index $d_{\mathbf{t}}(a, b)$ for each elementary criterion $g_{\mathbf{t}}$ :

$$
d_{\mathbf{t}}(a, b)= \begin{cases}0 & \text { if } g_{\mathbf{t}}(b)-g_{\mathbf{t}}(a) \leq p_{\mathbf{t}}, \\ \frac{\left[g_{\mathbf{t}}(b)-g_{\mathbf{t}}(a)\right]-p_{\mathbf{t}}}{v_{\mathbf{t}}-p_{\mathbf{t}}} & \text { if } p_{\mathbf{t}}<g_{\mathbf{t}}(b)-g_{\mathbf{t}}(a)<v_{\mathbf{t}} \\ 1 & \text { if } g_{\mathbf{t}}(b)-g_{\mathbf{t}}(a) \geq v_{\mathbf{t}} .\end{cases}
$$

It represents the degree of discordance with the hypothesis that $a$ outranks $b$ on elementary criterion $g_{\mathbf{t}}$. Differently from $\phi_{\mathbf{t}}(a, b)$, the partial discordance index is a non-decreasing function of the difference $g_{\mathbf{t}}(b)-g_{\mathbf{t}}(a)$.

- The partial concordance index $C_{\mathbf{r}}(a, b)$ for each criterion $G_{\mathbf{r}}, \mathbf{r} \in \mathcal{I}_{\mathcal{G}} \backslash E L$ :

$$
C_{\mathbf{r}}(a, b)=\sum_{\mathbf{t} \in E\left(G_{\mathbf{r}}\right)} w_{\mathbf{t}} \phi_{\mathbf{t}}(a, b) .
$$

It represents the degree of concordance with the hypothesis of outranking of $a$ over $b$ on criterion $G_{\mathbf{r}}$; it is computed taking into account simultaneously the preference of $a$ over $b$ on all the elementary criteria descending from criterion $G_{\mathbf{r}}$.

- The partial credibility index $\sigma_{\mathbf{r}}(a, b)$ for each criterion $G_{\mathbf{r}}, \mathbf{r} \in \mathcal{I}_{\mathcal{G}} \backslash E L$ :

$$
\sigma_{\mathbf{r}}(a, b)=C_{\mathbf{r}}(a, b) \prod_{\mathbf{t} \in \bar{F}_{\mathbf{r}}} \frac{1-d_{\mathbf{t}}(a, b)}{1-C_{\mathbf{r}}(a, b)}
$$

where $\bar{F}_{\mathbf{r}}=\left\{\mathbf{t} \in E\left(G_{\mathbf{r}}\right): d_{\mathbf{t}}(a, b)>C_{\mathbf{r}}(a, b)\right\}$.

It represents the credibility of the outranking of $a$ over $b$ on criterion $G_{\mathbf{r}}$, taking simultaneously into account all reasons in favor and all reasons against the preference of $a$ over $b$ on all elementary criteria descending from $G_{\mathbf{r}}$.

In order to design an assignment procedure of ELECRE-Tri type, we need, moreover, a cutting level $\lambda_{\mathbf{r}}$ on the credibility of outranking with respect to each criterion $G_{\mathbf{r}}$ in the hierarchy, except the elementary 
criteria.

In addition, two assumptions should be made:

1. $W_{\mathbf{r}}=\sum_{\mathbf{t} \in E\left(G_{\mathbf{r}}\right)} w_{\mathbf{t}}$, for each $\mathbf{r} \in \mathcal{I}_{\mathcal{G}}$; this means that the importance of criterion $G_{\mathbf{r}}$ in the hierarchy is obtained by adding up the importances of all the elementary criteria descending from criterion $G_{\mathbf{r}}$. As a consequence of this assumption, let us observe that $C_{\mathbf{r}}(a, b)=\sum_{j=1}^{n(\mathbf{r})} C_{(\mathbf{r}, j)}(a, b)$.

2. $\lambda_{\mathbf{r}} \in\left[W_{\mathbf{r}} / 2, W_{\mathbf{r}}\right]$, for all $\mathbf{r} \in \mathcal{I}_{\mathcal{G}} \backslash E L$.

On the basis of the above definitions, we can give the following three different outranking relations for each criterion $G_{\mathbf{r}}$ in the hierarchy:

O1) $a S_{\mathbf{r}}^{I} b \Leftrightarrow C_{\mathbf{r}}(a, b) \geq \lambda_{\mathbf{r}}$

O2) $a S_{\mathbf{r}}^{I I} b \Leftrightarrow C_{\mathbf{r}}(a, b) \geq \lambda_{\mathbf{r}}$ and $g_{\mathbf{t}}(b)-g_{\mathbf{t}}(a)<v_{\mathbf{t}}$, for all $\mathbf{t} \in E\left(G_{\mathbf{r}}\right)$,

O3) $a S_{\mathbf{r}}^{I I I} b \Leftrightarrow \sigma_{\mathbf{r}}(a, b) \geq \lambda_{\mathbf{r}}$.

The first outranking relation is based only on the reasons in favour of the outranking of $a$ over $b$, while the second and the third outranking relations are defined taking into account simultaneously the reasons in favor and the reasons against the outranking of $a$ over $b$ in two different ways.

Using any of the above outranking relations (here denoted generically by $S_{\mathbf{r}}$ ), we can define the following binary relations for each criterion $G_{\mathbf{r}}$ in the hierarchy:

- $a \succ_{\mathbf{r}} b\left(a\right.$ is preferred to $b$ on criterion $\left.G_{\mathbf{r}}\right)$ iff $a S_{\mathbf{r}} b$ and $\operatorname{not}\left(b S_{\mathbf{r}} a\right)$,

- $a \sim_{\mathbf{r}} b\left(a\right.$ is indifferent to $b$ on criterion $\left.G_{\mathbf{r}}\right)$ iff $a S_{\mathbf{r}} b$ and $b S_{\mathbf{r}} a$,

- $a ?_{\mathbf{r}} b$ ( $a$ is incomparable with $b$ on criterion $\left.G_{\mathbf{r}}\right)$ iff $\operatorname{not}\left(a S_{\mathbf{r}} b\right)$ and $\operatorname{not}\left(b S_{\mathbf{r}} a\right)$.

Note 2.2. Let us denote by $\left[C l_{h}, C l_{k}\right]$ the interval of contiguous categories going from $C l_{h}$ to $C l_{k}, 1 \leq h \leq$ $k \leq p$, that is $\left[C l_{h}, C l_{k}\right]=\left\{C l_{h}, C l_{h+1}, \ldots, C l_{k-1}, C l_{k}\right\}$. We suppose that the number and the semantics of the categories are the same for all criteria in the hierarchy, i.e., $\left\{C l_{1}, \ldots, C l_{p}\right\}$. In this way, we exclude the situation where an alternative could be assigned to interval of categories $\left[\mathrm{Cl}_{1}, C l_{p_{1}}\right]$ if we considered criterion $G_{\mathbf{r}_{1}}$, and to interval of categories $\left[C l_{1}, C l_{p_{2}}\right]$ if we considered criterion $G_{\mathbf{r}_{2}}$, with $p_{1} \neq p_{2}$.

\section{Coherence properties and conditions ensuring them}

Given a criterion $G_{\mathbf{r}}, \mathbf{r} \in \mathcal{I}_{\mathcal{G}} \backslash\{E L \cup L B O\}$, we impose two coherence properties:

C1) if $a S_{(\mathbf{r}, j)} b, j=1, \ldots, n(\mathbf{r})$, then $a S_{\mathbf{r}} b$,

C2) if $\operatorname{not}\left(a S_{(\mathbf{r}, j)} b\right), j=1, \ldots, n(\mathbf{r})$, then $\operatorname{not}\left(a S_{\mathbf{r}} b\right)$. 
Coherence property $\mathbf{C 1}$ ) states that if $a$ outranks $b$ on all subcriteria of $G_{\mathbf{r}}$ in the subsequent level, then $a$ should outrank $b$ on criterion $G_{\mathbf{r}}$, while coherence property C2) states that if $a$ does not outrank $b$ for all subcriteria of $G_{\mathbf{r}}$ in the subsequent level, then $a$ should not outrank $b$ also on criterion $G_{\mathbf{r}}$. To motivate this property, let us suppose that we compare students $a$ and $b$ with respect to mathematics, and that algebra and geometry are direct subcriteria of mathematics. Coherence property C1) ensures that if student $a$ outranks student $b$ on algebra and geometry, then $a$ outranks $b$ also on mathematics; analogously, coherence property C2) ensures that if $a$ does not outrank $b$ neither on algebra nor on geometry, then $a$ does not outrank $b$ on mathematics.

Considering the outranking relations O1) and O2), we look for some conditions on the parameters of the model and, in particular, on the cutting levels, such that coherence properties C1) and C2) are satisfied. Theorem 3.1 provides necessary and sufficient conditions ensuring that the coherence properties C1) and C2) hold.

Theorem 3.1. Let $G_{\mathbf{r}}, \mathbf{r} \in \mathcal{I}_{\mathcal{G}} \backslash\{E L \cup L B O\}$ and let us define, for each $j=1, \ldots, n(\mathbf{r})$,

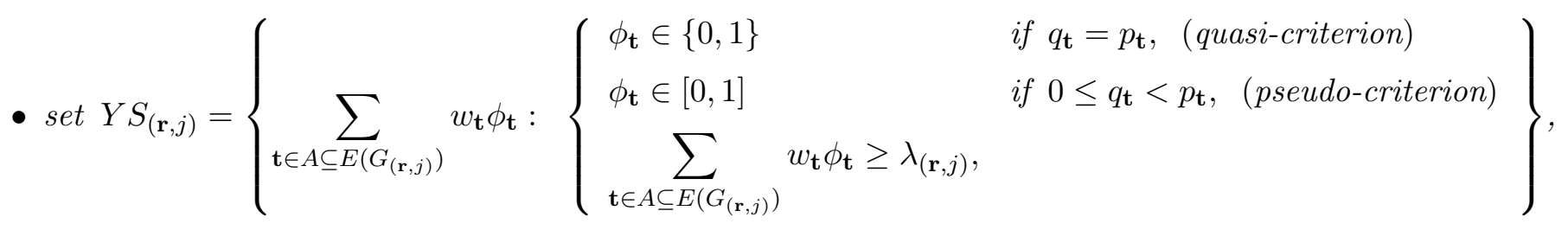

- $m_{(\mathbf{r}, j)}=\min Y S_{(\mathbf{r}, j)}$,

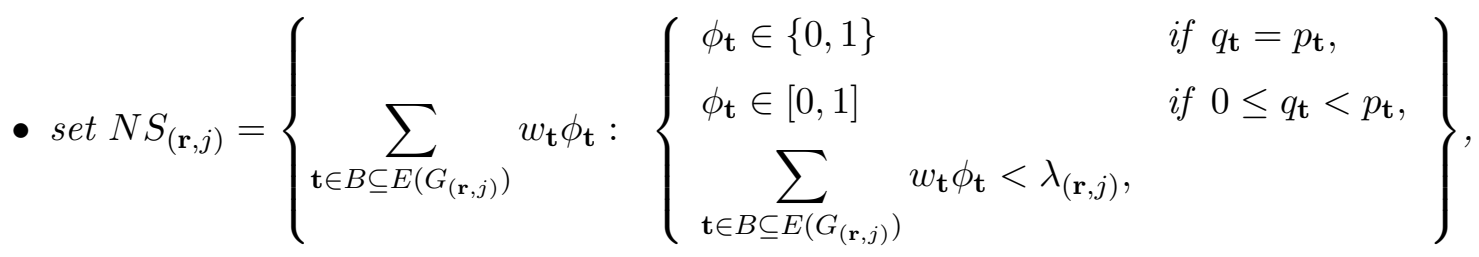

- $M_{(\mathbf{r}, j)}=\sup N S_{(\mathbf{r}, j)}$,

- $m_{\mathbf{r}}=\sum_{j=1}^{n(\mathbf{r})} m_{(\mathbf{r}, j)}$, and $M_{\mathbf{r}}=\sum_{j=1}^{n(\mathbf{r})} M_{(\mathbf{r}, j)}$,

then properties $\mathbf{C 1}$ ) and $\mathbf{C 2})$ hold if and only if

$$
\begin{cases}M_{\mathbf{r}}<\lambda_{\mathbf{r}} \leq m_{\mathbf{r}} & \text { if } M_{(\mathbf{r}, j)}=\max N S_{(\mathbf{r}, j)}, \text { for all } j=1, \ldots, n(\mathbf{r}), \\ M_{\mathbf{r}} \leq \lambda_{\mathbf{r}} \leq m_{\mathbf{r}} & \text { if } M_{(\mathbf{r}, j)}=\sup N S_{(\mathbf{r}, j)}=\lambda_{(\mathbf{r}, j)}, \text { for at least one } j \in\{1, \ldots, n(\mathbf{r})\}\end{cases}
$$

Theorem 3.1 can be explained in the following way: let $G_{\mathbf{r}}$ a criterion in the hierarchy having $G_{(\mathbf{r}, j)}$, $j=1, \ldots, n(\mathbf{r})$, as direct subcriteria, and let $\lambda_{(\mathbf{r}, j)}$ the cutting level associated with criterion $G_{(\mathbf{r}, j)}, j=$ $1, \ldots, n(\mathbf{r})$. Computing for each criterion $G_{(\mathbf{r}, j)}$ the minimum of the set of the reachable concordance indices $C_{(\mathbf{r}, j)}$ being not smaller than the cutting level $\lambda_{(\mathbf{r}, j)}$, that is $m_{(\mathbf{r}, j)}$, and the supremum of the set of the reachable concordance indices $C_{(\mathbf{r}, j)}$ being smaller than the corresponding cutting level $\lambda_{(\mathbf{r}, j)}$, that is $M_{(\mathbf{r}, j)}$, 
if the cutting level $\lambda_{\mathbf{r}}$ is in the interval of extremes $\sum_{j=1}^{n(\mathbf{r})} M_{(\mathbf{r}, j)}$ and $\sum_{j=1}^{n(\mathbf{r})} m_{(\mathbf{r}, j)}$, then coherence properties $\mathbf{C 1 )}$ and C2) are satisfied, and viceversa. On one hand, $\sum_{j=1}^{n(\mathbf{r})} M_{(\mathbf{r}, j)}$ can be interpreted as the maximum value of the concordance index $C_{\mathbf{r}}(a, b)$, such that $\operatorname{not}\left(a S_{(r, j)} b\right)$ holds for all $j=1, \ldots, n(\mathbf{r})$; this means that considering a value of the concordance index $C_{\mathbf{r}}(a, b)$ greater than $M_{(\mathbf{r}, j)}$ implies that $a S_{(\mathbf{r}, j)} b$ holds for at least one $j \in\{1, \ldots, n(\mathbf{r})\}$; on the other hand, $\sum_{j=1}^{n(\mathbf{r})} m_{(\mathbf{r}, j)}$ can be interpreted as the minimum value of the concordance index $C_{\mathbf{r}}(a, b)$, such that $a S_{(r, j)} b$ holds for all $j=1, \ldots, n(\mathbf{r})$; this means that considering a value of the concordance index $C_{\mathbf{r}}(a, b)$ smaller than $m_{(\mathbf{r}, j)}, \operatorname{not}\left(a S_{(\mathbf{r}, j)} b\right)$ holds for at least one $j \in\{1, \ldots, n(\mathbf{r})\}$.

Let us observe that Theorem 3.1 is really important, not only because it provides necessary and sufficient conditions on the cutting levels, such that coherence properties C1) and C2) are satisfied, but also because it provides a hint on how to fix the cutting level $\lambda_{\mathbf{r}}$ of criterion $G_{\mathbf{r}}$ knowing the cutting levels $\lambda_{(\mathbf{r}, j)}$ of all direct subcriteria $G_{(\mathbf{r}, j)}, j=1, \ldots, n(\mathbf{r})$, of criterion $G_{\mathbf{r}}$.

Note 3.1. It is meaningful to observe that $m_{(\mathbf{r}, j)}$ could be different from $\lambda_{(\mathbf{r}, j)}$ and that the presence of at least one pseudo-criterion among the elementary criteria of $G_{\mathbf{r}}$ does not ensure that the value $\lambda_{(\mathbf{r}, j)}$ could be reached. For example, let us consider $E\left(G_{(\mathbf{r}, 1)}\right)=\left\{g_{(\mathbf{r}, 1,1)}, g_{(\mathbf{r}, 1,2)}, g_{(\mathbf{r}, 1,3)}\right\}$ having $w_{(\mathbf{r}, 1,1)}=0.4$, $w_{(\mathbf{r}, 1,2)}=0.4$ and $w_{(\mathbf{r}, 1,3)}=0.2$ as weights, and that $g_{(\mathbf{r}, 1,1)}$ and $g_{(\mathbf{r}, 1,2)}$ are quasi-criteria, while $g_{(\mathbf{r}, 1,3)}$ is a pseudo-criterion. This implies that $w_{\mathbf{t}} \phi_{\mathbf{t}} \in\{0,0.4\}, \mathbf{t}=(\mathbf{r}, 1,1),(\mathbf{r}, 1,2)$ and $w_{(\mathbf{r}, 1,3)} \phi_{(\mathbf{r}, 1,3)} \in[0,0.2]$. As a consequence

$$
\sum_{i=1}^{3} w_{(\mathbf{r}, 1, i)} \phi_{(\mathbf{r}, 1, i)} \in \begin{cases}{[0,0.2]} & \text { if } \phi_{1}=\phi_{2}=0 \\ {[0.4,0.6]} & \text { if } \phi_{1}=1 \text { and } \phi_{2}=0 \text { or viceversa }, \\ {[0.8,1]} & \text { if } \phi_{1}=\phi_{2}=1 .\end{cases}
$$

This means that a cutting level $\left.\lambda_{(\mathbf{r}, 1)} \in\right] 0.6,0.8[$ can never be reached (analogously for a hypothetical cutting level belonging to ]0.2, 0.4[, even if this level is non-acceptable for the hypothesis that it should be not smaller than a half of the sum of weights of the considered criteria, and, therefore, at least equal to 0.5).

Let us observe that, if we fixed $\lambda_{(\mathbf{r}, 1)}=0.7$, then $m_{(\mathbf{r}, 1)}=0.8$, while if we fixed $\lambda_{(\mathbf{r}, 1)}=0.9$, then $m_{(\mathbf{r}, 1)}=0.9$ because this value can be reached.

To be yet more precise, let us also observe the difference between cases $\left(^{*}\right)$ and $\left(^{* *}\right)$.

If we set $\lambda_{(\mathbf{r}, 1)}=0.7$, then $M_{(\mathbf{r}, 1)}=0.6$ being the maximum of $N S_{(\mathbf{r}, 1)}$. If, instead, we set $\lambda_{(\mathbf{r}, 1)}=0.55$, then $M_{(\mathbf{r}, 1)}=0.55$ is a supremum of $N S_{(\mathbf{r}, 1)}$ but not a maximum.

Note 3.2. Looking at Theorem 3.1, one can ask why the two coherence properties do not involve any condition on the veto thresholds while the definition of outranking relation $\mathbf{O} 2)$ involves the veto thresholds. The reason is the following. Noting that for each criterion $G_{\mathbf{r}}, E\left(G_{\mathbf{r}}\right)=\cup_{j=1}^{n(\mathbf{r})} E\left(G_{(\mathbf{r}, j)}\right)$, considering two alternatives $a, b \in A$, if a outranks $b$ on $G_{(\mathbf{r}, j)}, j=1, \ldots, n(\mathbf{r})$, then no elementary criterion descending from $G_{(\mathbf{r}, j)}$ opposes a veto to the ouranking of a over $b$ on $G_{(\mathbf{r}, j)}$; as a consequence, no elementary criterion descending from $E\left(G_{\mathbf{r}}\right)$ opposes a veto to the outranking of a over $b$ on criterion $G_{\mathbf{r}}$. Formally,

- if $a S_{(\mathbf{r}, j)} b$ for all $j=1, \ldots, n(\mathbf{r})$, then $g_{\mathbf{t}}(b)-g_{\mathbf{t}}(a)<v_{\mathbf{t}}$ for all $\mathbf{t} \in E\left(G_{(\mathbf{r}, j)}\right)$ and, consequently, $g_{\mathbf{t}}(b)-g_{\mathbf{t}}(a)<v_{\mathbf{t}}$ for all $\mathbf{t} \in E\left(G_{\mathbf{r}}\right)$, implying that $a S_{\mathbf{r}} b$.

Analogously, if a does not outrank $b$ on $G_{(\mathbf{r}, j)}, j \in\{1, \ldots, n(\mathbf{r})\}$, because there is one elementary criterion descending from $G_{(\mathbf{r}, j)}$ opposing a veto to the outranking of a over $b$ on $G_{(\mathbf{r}, j)}$, then the same elementary criterion, being an elementary criterion of $G_{\mathbf{r}}$, will also oppose a veto to the outranking of a over $b$ on $G_{\mathbf{r}}$. Formally, supposing that $C_{(\mathbf{r}, j)}(a, b) \geq \lambda_{(\mathbf{r}, j)}$ for all $j=1, \ldots, n(\mathbf{r})$, 
- if $\operatorname{not}\left(a S_{(\mathbf{r}, j)} b\right)$ for some $j \in\{1, \ldots, n(\mathbf{r})\}$, then $g_{\mathbf{t}}(b)-g_{\mathbf{t}}(a) \geq v_{\mathbf{t}}$ for at least one $\mathbf{t} \in E\left(G_{(\mathbf{r}, j)}\right)$ and, consequently, $g_{\mathbf{t}}(b)-g_{\mathbf{t}}(a) \geq v_{\mathbf{t}}$ for at least one $\mathbf{t} \in E\left(G_{\mathbf{r}}\right)$, implying $\operatorname{not}\left(a S_{\mathbf{r}} b\right)$.

This is the reason for which Theorem 3.1 and, therefore, Corollaries 3.1 and 3.2 do not put any restriction on the veto thresholds but on the cutting levels only.

Proof. of Theorem 3.1.

$(\Rightarrow)$ Let us prove the thesis in case $(*)$.

Let us suppose, for contradiction, that $M_{\mathbf{r}} \geq \lambda_{\mathbf{r}}$, and let $a, b \in A$ such that $C_{(\mathbf{r}, j)}(a, b)=M_{(\mathbf{r}, j)}$ for all $j=1, \ldots, n(\mathbf{r})$. This implies $\operatorname{not}\left(a S_{(\mathbf{r}, j)} b\right)$ for all $j=1, \ldots, n(\mathbf{r})$. Adding up with respect to the $j$ index, we have $C_{\mathbf{r}}(a, b)=\sum_{j=1}^{n(\mathbf{r})} M_{(\mathbf{r}, j)}=M_{\mathbf{r}} \geq \lambda_{\mathbf{r}}$ and, consequently, $a S_{\mathbf{r}} b$, which contradicts the coherence property C2).

Let us prove the thesis in case $(* *)$.

Let us suppose, for contradiction, that $M_{\mathbf{r}}>\lambda_{\mathbf{r}}$ and, let us consider $a, b \in A$ such that $C_{(\mathbf{r}, j)}(a, b)=M_{(\mathbf{r}, j)}-\varepsilon$ for all $j=1, \ldots, n(\mathbf{r})$ with $\varepsilon>0$. In this case, since $M_{(\mathbf{r}, j)} \leq \lambda_{(\mathbf{r}, j)}$, we shall have $\operatorname{not}\left(a S_{(\mathbf{r}, j)} b\right)$ for all $j=1, \ldots, n(\mathbf{r})$. Adding up with respect to $j$, we get $C_{\mathbf{r}}(a, b)=\sum_{j=1}^{n(\mathbf{r})} M_{(\mathbf{r}, j)}-n(\mathbf{r}) \times \varepsilon=M_{\mathbf{r}}-n(\mathbf{r}) \times \varepsilon$. Then, setting $0<\varepsilon<\frac{M_{\mathbf{r}}-\lambda_{\mathbf{r}}}{n(\mathbf{r})}$, we shall get that $C_{\mathbf{r}}(a, b)>\lambda_{\mathbf{r}}$, which contradicts the coherence property $\left.\mathbf{C 2}\right)$.

$(\Leftarrow)$ Let $a, b \in A$ such that $a S_{(\mathbf{r}, j)} b$ for all $j=1, \ldots, n(\mathbf{r})$. This implies that $C_{(\mathbf{r}, j)}(a, b) \geq \lambda_{(\mathbf{r}, j)}$ for all $j=1, \ldots, n(\mathbf{r})$. Consequently, $C_{(\mathbf{r}, j)}(a, b) \in Y S_{(\mathbf{r}, j)}$ and $C_{(\mathbf{r}, j)}(a, b) \geq m_{(\mathbf{r}, j)}$ for all $j=1, \ldots, n(\mathbf{r})$. Adding up with respect to $j$, we get $C_{\mathbf{r}}(a, b)=\sum_{j=1}^{n(\mathbf{r})} C_{(\mathbf{r}, j)}(a, b) \geq \sum_{j=1}^{n(\mathbf{r})} m_{(\mathbf{r}, j)}=m_{\mathbf{r}} \geq \lambda_{\mathbf{r}}$ and, therefore, $C_{\mathbf{r}}(a, b) \geq \lambda_{\mathbf{r}}$ implying that $a S_{\mathbf{r}} b$ being coherence property $\mathbf{C 1}$ ).

In a first moment, let us prove that coherence property C2) holds under hypothesis $(*)$.

Let $a, b \in A$ be such that $\operatorname{not}\left(a S_{(\mathbf{r}, j)} b\right)$ for all $j=1, \ldots, n(\mathbf{r})$. This implies that $C_{(\mathbf{r}, j)}(a, b)<\lambda_{(\mathbf{r}, j)}$ for all $j=$ $1, \ldots, n(\mathbf{r})$ and, consequently, $C_{(\mathbf{r}, j)}(a, b) \in N S_{(\mathbf{r}, j)}$ and $C_{(\mathbf{r}, j)}(a, b) \leq M_{(\mathbf{r}, j)}$ for all $j=1, \ldots, n(\mathbf{r})$. Adding up with respect to $j$, we get $C_{\mathbf{r}}(a, b)=\sum_{j=1}^{n(\mathbf{r})} C_{(\mathbf{r}, j)}(a, b) \leq \sum_{j=1}^{n(\mathbf{r})} M_{(\mathbf{r}, j)}=M_{\mathbf{r}}<\lambda_{\mathbf{r}}$. Therefore, $C_{\mathbf{r}}(a, b)<\lambda_{\mathbf{r}}$ and, as a consequence, $\operatorname{not}\left(a S_{\mathbf{r}} b\right)$.

Now, let us prove that coherence property C2) holds under hypothesis $(* *)$.

Let $a, b \in A$ be such that $\operatorname{not}\left(a S_{(\mathbf{r}, j)} b\right)$ for all $j=1, \ldots, n(\mathbf{r})$. This implies that $C_{(\mathbf{r}, j)}(a, b)<\lambda_{(\mathbf{r}, j)}$ for all $j=1, \ldots, n(\mathbf{r})$ and, consequently, $C_{(\mathbf{r}, j)}(a, b) \in N S_{(\mathbf{r}, j)}$. Since for some $j, M_{(\mathbf{r}, j)}=\sup N S_{(\mathbf{r}, j)}$ but it is not a maximum, then, for such $j$ we shall have $C_{(\mathbf{r}, j)}<M_{(\mathbf{r}, j)}$. As a consequence, adding up with respect to $j$, we get $C_{\mathbf{r}}(a, b)=\sum_{j=1}^{n(\mathbf{r})} C_{(\mathbf{r}, j)}(a, b)<\sum_{j=1}^{n(\mathbf{r})} M_{(\mathbf{r}, j)}=M_{\mathbf{r}} \leq \lambda_{\mathbf{r}}$. Therefore, $C_{\mathbf{r}}(a, b)<\lambda_{\mathbf{r}}$ and, as a consequence, $\operatorname{not}\left(a S_{\mathbf{r}} b\right)$ being coherence property $\left.\mathbf{C 2}\right)$.

Two Corollaries follow from Theorem 3.1. They correspond to the case where all elementary criteria are quasi-criteria, and to the case where all elementary criteria are pseudo-criteria, respectively.

Corollary 3.1. Let us suppose that all elementary criteria are quasi-criteria $\left(q_{\mathbf{t}}=p_{\mathbf{t}}\right.$ for all $\left.\mathbf{t} \in E L\right)$ and let give the following definitions for each $G_{\mathbf{r}}, \mathbf{r} \in \mathcal{I}_{\mathcal{G}} \backslash\{E L \cup L B O\}$ and, for each $j=1, \ldots, n(\mathbf{r})$ :

- set $Y S_{(\mathbf{r}, j)}=\left\{\sum_{\mathbf{t} \in A \subseteq E\left(G_{(\mathbf{r}, j)}\right)} w_{\mathbf{t}}: \sum_{\mathbf{t} \in A \subseteq E\left(G_{(\mathbf{r}, j)}\right)} w_{\mathbf{t}} \geq \lambda_{(\mathbf{r}, j)}\right\}$, 
- $m_{(\mathbf{r}, j)}=\min Y S_{(\mathbf{r}, j)}$,

- $\operatorname{set} N S_{(\mathbf{r}, j)}=\left\{\sum_{\mathbf{t} \in B \subseteq E\left(G_{(\mathbf{r}, j)}\right)} w_{\mathbf{t}}: \sum_{\mathbf{t} \in B \subseteq E\left(G_{(\mathbf{r}, j)}\right)} w_{\mathbf{t}}<\lambda_{(\mathbf{r}, j)}\right\}$,

- $M_{(\mathbf{r}, j)}=\max N S_{(\mathbf{r}, j)}$,

- $m_{\mathbf{r}}=\sum_{j=1}^{n(\mathbf{r})} m_{(\mathbf{r}, j)}$, and $M_{\mathbf{r}}=\sum_{j=1}^{n(\mathbf{r})} M_{(\mathbf{r}, j)}$,

then coherence properties C1) and C2) hold if and only if $M_{\mathbf{r}}<\lambda_{\mathbf{r}} \leq m_{\mathbf{r}}$.

Proof. Two things should be noted in this case:

1. Since all elementary criteria are quasi-criteria, the partial concordance index can only get value 0 or 1 . Therefore, in sets $Y S_{(\mathbf{r}, j)}$ (analogously, in sets $N S_{(\mathbf{r}, j)}$ ), we have to take into account the coalitions of elementary criteria descending from $G_{(\mathbf{r}, j)}$ such that the sum of the importance coefficients is at least equal (smaller than) the cutting level $\lambda_{(\mathbf{r}, j)}$.

2. We have to consider case $(*)$ only, because in this case, $N S_{(\mathbf{r}, j)}$ is composed of a finite number of elements and, consequently, it has always a maximum.

Corollary 3.2. If all elementary criteria are pseudo-criteria $\left(0 \leq q_{\mathbf{t}}<p_{\mathbf{t}}\right.$ for all $\left.\mathbf{t} \in E L\right)$, then coherence properties $\boldsymbol{C 1}$ ) and $\boldsymbol{C 2})$ hold if and only if $\lambda_{\mathbf{r}}=\sum_{j=1}^{n(\mathbf{r})} \lambda_{(\mathbf{r}, j)}$ for all $\mathbf{r} \in \mathcal{I}_{\mathcal{G}} \backslash\{E L \cup L B O\}$.

Proof. It is sufficient to observe that in this case, $\sum_{\mathbf{t} \in A \subseteq E\left(G_{(\mathbf{r}, j)}\right)} w_{\mathbf{t}} \phi_{\mathbf{t}}$ could take all values in the interval $\left[0, W_{(\mathbf{r}, j)}\right]$ and, in consequence,

$$
m_{(\mathbf{r}, j)}=\lambda_{(\mathbf{r}, j)}=M_{(\mathbf{r}, j)} \text { for all } j=1, \ldots, n(\mathbf{r}) .
$$

By equation (5) we get $m_{\mathbf{r}}=\sum_{j=1}^{n(\mathbf{r})} m_{(\mathbf{r}, j)}=\sum_{j=1}^{n(\mathbf{r})} \lambda_{(\mathbf{r}, j)}$ and $M_{\mathbf{r}}=\sum_{j=1}^{n(\mathbf{r})} M_{(\mathbf{r}, j)}=\sum_{j=1}^{n(\mathbf{r})} \lambda_{(\mathbf{r}, j)}$, and since in this case the hypothesis $(* *)$ holds, we get the thesis.

In the following, we shall suppose without loss of generality that all elementary criteria are pseudo-criteria and that coherence properties C1) and C2) are satisfied. From Corollary 3.2 it follows that $\lambda_{\mathbf{r}}=\sum_{j=1}^{n(\mathbf{r})} \lambda_{(\mathbf{r}, j)}$ for all $G_{\mathbf{r}}, \mathbf{r} \in \mathcal{I}_{\mathcal{G}} \backslash E L$ and, consequently, one needs to define the cutting level $\lambda$ only for the subcriteria located at the last but one level of the hierarchy, that is subcriteria $G_{\mathbf{s}}, \mathbf{s} \in L B O$. In fact, from this it obviously follows that $\lambda_{\mathbf{r}}=\sum_{\mathbf{s} \in L B\left(G_{\mathbf{r}}\right)} \lambda_{\mathbf{s}}$ for each $G_{\mathbf{r}}, \mathbf{r} \in \mathcal{I}_{\mathcal{G}} \backslash E L$.

Note 3.3. Let us observe that Theorem 3.1, as well as Corollaries 3.1 and 3.2, do not hold for hierarchical versions of ELECTRE Tri sorting methods only, but also for hierarchical versions of other ELECTRE methods dealing with ranking and choice problems. In general, the coherence properties C1) and C2) hold for the outranking relation considered in a hierarchical setting. 


\section{MCHP and the ELECTRE Tri methods}

In this section, we shall apply MCHP to the three considered ELECTRE Tri methods, and we shall explain how to extend the concordance indices previously defined to the case of interacting elementary criteria.

\subsection{MCHP applied to the ELECTRE Tri-B method}

Given an alternative $a \in A$ and a criterion $G_{\mathbf{r}}, \mathbf{r} \in \mathcal{I}_{\mathcal{G}} \backslash E L$, two different assignment procedures can be defined:

- Pessimistic assignment:

- compare successively $a$ to $b_{h}$ on criterion $G_{\mathbf{r}}$, for $h=p, p-1, \ldots, 0$,

$-b_{l}$ being the first reference profile such that $a S_{\mathbf{r}} b_{l}$, assign $a$ to category $C l_{l+1}$ (we shall write $\left.a \underset{\mathbf{r}}{\rightarrow} C l_{l+1}\right)$.

- Optimistic assignment:

- compare $a$ successively to $b_{h}$ on criterion $G_{\mathbf{r}}$, for $h=1,2, \ldots, p$,

$-b_{l}$ being the first reference profile such that $b_{l} \succ_{\mathbf{r}} a$, assign $a$ to category $C l_{l}$.

Let us precise that $b_{0}$ and $b_{p}$ are two extreme profiles, such that $a \succ_{\mathbf{r}} b_{0}$, and $b_{p} \succ_{\mathbf{r}} a$ for all $a \in A$, and for all criteria $G_{\mathbf{r}}, \mathbf{r} \in \mathcal{I}_{\mathcal{G}} \backslash E L$.

Definition 4.1. Given $a, b \in A$ and $G_{\mathbf{r}}, \mathbf{r} \in \mathcal{I}_{\mathcal{G}} \backslash E L$, we say that:

- a dominates $b$ iff $g_{\mathbf{t}}(a) \geq g_{\mathbf{t}}(b)$, for all $\mathbf{t} \in E L$, and there exists at least one $\mathbf{t}_{1} \in E L$ such that $g_{\mathbf{t}_{1}}(a)>g_{\mathbf{t}_{1}}(b)$,

- a dominates $b$ with respect to criterion $G_{\mathbf{r}}$ if $g_{\mathbf{t}}(a) \geq g_{\mathbf{t}}(b)$, for all $\mathbf{t} \in E\left(G_{\mathbf{r}}\right)$, and there exists at least one $\mathbf{t}_{1} \in E\left(G_{\mathbf{r}}\right)$ such that $g_{\mathbf{t}_{1}}(a)>g_{\mathbf{t}_{1}}(b)$.

Considering the first two outranking relations (O1) and O2)) defined above, we give a Lemma to prove Theorem 4.1:

Lemma 4.1. Given $a, b, c \in A$ and the criterion $G_{\mathbf{r}}, \mathbf{r} \in \mathcal{I}_{\mathcal{G}}$,

1) if a dominates $b$ with respect to criterion $G_{\mathbf{r}}$ and $b S_{\mathbf{r}} c$, then $a S_{\mathbf{r}} c$,

2) if $a S_{\mathbf{r}} b$ and $b$ dominates $c$ with respect to criterion $G_{\mathbf{r}}$, then $a S_{\mathbf{r}} c$,

3) if c dominates a with respect to criterion $G_{\mathbf{r}}$ and $\operatorname{not}\left(c S_{\mathbf{r}} b\right)$, then $\operatorname{not}\left(a S_{\mathbf{r}} b\right)$,

4) if $\operatorname{not}\left(a S_{\mathbf{r}} b\right)$ and $c$ dominates $b$ with respect to criterion $G_{\mathbf{r}}$, then $\operatorname{not}\left(a S_{\mathbf{r}} c\right)$.

Proof. See Appendix A.

Applying MCHP to ELECTRE Tri-B, we get the following theorem: 
Theorem 4.1. Given alternative $a \in A$, and criterion $G_{\mathbf{r}}, \mathbf{r} \in \mathcal{I}_{\mathcal{G}} \backslash E L$, the following implication holds:

$$
a \underset{(\mathbf{r}, 1)}{\longrightarrow} C l_{k_{1}}, a \underset{(\mathbf{r}, 2)}{\longrightarrow} C l_{k_{2}}, \ldots, a \underset{(\mathbf{r}, n(\mathbf{r}))}{\longrightarrow} C l_{k_{n(\mathbf{r})}} \Rightarrow a \underset{\mathbf{r}}{\longrightarrow} C l_{k}
$$

where

- $\min \left\{k_{1}, k_{2}, \ldots, k_{n(\mathbf{r})}\right\} \leq k \leq \max \left\{k_{1}, k_{2}, \ldots, k_{n(\mathbf{r})}\right\}$ if we consider the pessimistic assignment,

- $k \leq \max \left\{k_{1}, k_{2}, \ldots, k_{n(\mathbf{r})}\right\}$ if we consider the optimistic assignment.

Proof. See Appendix A.

Note 4.1. We note that in the optimistic assignment it is possible that alternative a is assigned to category $C l_{k}$ with $k<\min \left\{k_{1}, \ldots, k_{n(\mathbf{r})}\right\}$ because, in general, $\operatorname{not}\left(b_{h} \succ_{(\mathbf{r}, j)}\right.$ a) for all $j=1, \ldots, n(\mathbf{r})$ does not imply not $\left(b_{h} \succ_{\mathbf{r}} a\right)$. This means that it is possible that $b_{h}$ is not preferred to a for all direct subcriteria of criterion $G_{\mathbf{r}}$, but $b_{h}$ is preferred to a on $G_{\mathbf{r}}$. In consequence, even if alternative a is assigned to category $C l_{h+1}$ by all subcriteria $G_{\mathbf{r}}$, it is assigned to category $C_{h}$ at the aggregate level by criterion $G_{\mathbf{r}}$.

The following example confirms and explains the strange situation observed in Note 4.1 due to the property of the optimistic classification procedure proposed by the authors of ELECTRE Tri.

Inspired by [5], let us suppose that a USA company decides to open an office in Europe and, for this reason, the managing director has to decide the best location for it. Each possible location is characterized by two main factors that are business potential $\left(G_{\mathbf{1}}\right)$ and staffing issues $\left(G_{\mathbf{2}}\right)$; in particular, the public sector business potential $\left(g_{(\mathbf{1}, 1)}\right)$ and the private sector business potential $\left(g_{(\mathbf{1}, 2)}\right)$ are elementary criteria of $G_{\mathbf{1}}$, while the attractiveness to USA staff $\left(g_{(\mathbf{2}, 1)}\right)$ and the availability of staff $\left(g_{(\mathbf{2}, 2)}\right)$ are elementary criteria of $G_{\mathbf{2}}$. The hierarchy of the considered criteria is shown in Figure 1.

Figure 1: Hierarchy of criteria for the considered location problem

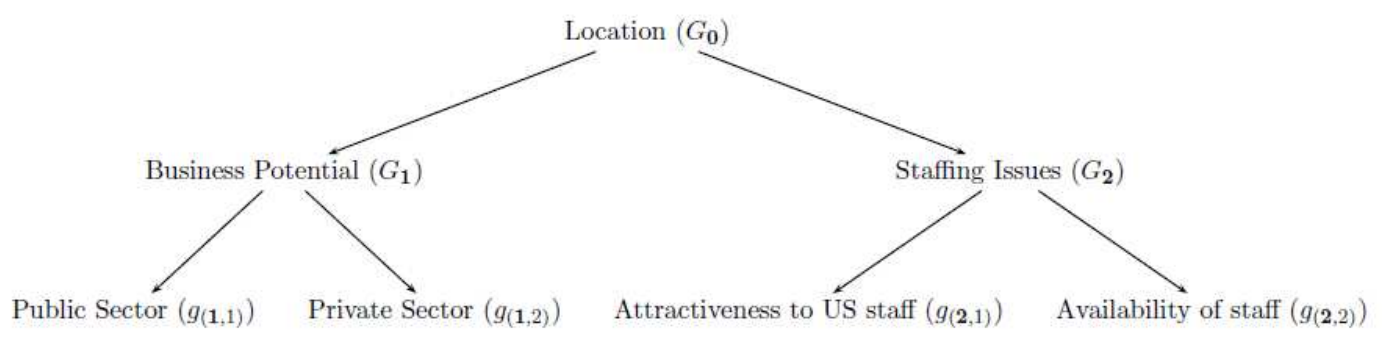

Let us suppose that each considered location has to be assigned to one of the following categories of suitability: "Bad" $\left(\mathrm{Cl}_{1}\right)$, "Medium" $\left(\mathrm{Cl}_{2}\right)$ and "Good" $\left(\mathrm{Cl}_{3}\right)$. Let us also focus on the optimistic assignment of a single location $a$ with respect to criteria $G_{\mathbf{1}}$ and $G_{\mathbf{2}}$, as well as with respect to the comprehensive level $G_{\mathbf{0}}$. The evaluations of $a$ and those of the reference profiles $b_{1}$ and $b_{2}$ separating the categories "Bad-Medium" and "Medium-Good", respectively, are provided in Table 1(a). The elementary criteria are supposed to be equally important and, therefore, the weight assigned to each elementary criterion is equal to 0.25 . Moreover, the indifference and preference thresholds are fixed to be 1 and 3, respectively, for all elementary criteria, 
while the cutting levels for subcriteria $G_{1}$ and $G_{\mathbf{2}}$ are set to $\lambda_{\mathbf{1}}=\lambda_{\mathbf{2}}=0.35$ and, consequently, the cutting level for criterion $G_{\mathbf{0}}$ is set to $\lambda_{\mathbf{0}}=0.7$. No veto threshold is assumed for any elementary criterion.

Table 1: Evaluations and partial concordance indices with respect to all criteria in the hierarchy

(a) Evaluations of the location and of the reference profiles

\begin{tabular}{|c|cc|cc|}
\hline & $g_{(\mathbf{1}, 1)}$ & $g_{(\mathbf{1}, 2)}$ & $g_{(\mathbf{2}, 1)}$ & $g_{(\mathbf{2}, 2)}$ \\
\hline$a$ & 3 & 1.2 & 4 & 1 \\
$b_{1}$ & 2 & 3 & 1 & 4 \\
$b_{2}$ & 5 & 6 & 4 & 7 \\
\hline
\end{tabular}

(c) Partial concordance index with respect to criterion $G_{\mathbf{2}}$

\begin{tabular}{|c|ccc|}
\hline$C_{\mathbf{2}}(\cdot, \cdot)$ & $a$ & $b_{1}$ & $b_{2}$ \\
\hline$a$ & 0.5 & 0.25 & 0.25 \\
$b_{1}$ & 0.25 & 0.5 & 0 \\
$b_{2}$ & 0.5 & 0.5 & 0.5 \\
\hline
\end{tabular}

(b) Partial concordance index with respect to criterion $G_{1}$

\begin{tabular}{|c|ccc|}
\hline$C_{\mathbf{1}}(\cdot, \cdot)$ & $a$ & $b_{1}$ & $b_{2}$ \\
\hline$a$ & 0.5 & 0.4 & 0.175 \\
$b_{1}$ & 0.5 & 0.5 & 0 \\
$b_{2}$ & 0.5 & 0.5 & 0.5 \\
\hline
\end{tabular}

(d) Partial concordance index with respect to criterion $G_{0}$

\begin{tabular}{|c|ccc|}
\hline$C_{\mathbf{0}}(\cdot, \cdot)$ & $a$ & $b_{1}$ & $b_{2}$ \\
\hline$a$ & 1 & 0.65 & 0.425 \\
$b_{1}$ & 0.75 & 1 & 0 \\
$b_{2}$ & 1 & 1 & 1 \\
\hline
\end{tabular}

Looking at Table 1(b), one can observe that

a) $C_{\mathbf{1}}\left(a, b_{1}\right)=0.4 \geq 0.35=\lambda_{\mathbf{1}} \Rightarrow a S_{\mathbf{1}} b_{1}$, and $C_{\mathbf{1}}\left(b_{1}, a\right)=0.5 \geq 0.35=\lambda_{\mathbf{1}} \Rightarrow b_{1} S_{\mathbf{1}} a$,

B) $C_{\mathbf{1}}\left(a, b_{2}\right)=0.175<0.35=\lambda_{\mathbf{1}} \Rightarrow \operatorname{not}\left(a S_{\mathbf{1}} b_{2}\right)$, and $C_{\mathbf{1}}\left(b_{2}, a\right)=0.5 \geq 0.35=\lambda_{\mathbf{1}} \Rightarrow b_{2} S_{\mathbf{1}} a$.

For $\alpha$ ), $a \sim_{\mathbf{1}} b_{1}$, while for $\beta$ ), $b_{2} \succ_{\mathbf{1}} a$. Consequently, the optimistic procedure applied in $G_{\mathbf{1}}$ will make the following assignment: $a \underset{\mathbf{1}}{\rightarrow} C l_{2}$.

Looking at Table 1(c), one can observe that

r) $C_{\mathbf{2}}\left(a, b_{1}\right)=0.25<0.35=\lambda_{\mathbf{2}} \Rightarrow \operatorname{not}\left(a S_{\mathbf{2}} b_{1}\right)$, and $C_{\mathbf{2}}\left(b_{1}, a\right)=0.25<0.35=\lambda_{\mathbf{2}} \Rightarrow \operatorname{not}\left(b_{1} S_{\mathbf{2}} a\right)$,

б) $C_{\mathbf{2}}\left(a, b_{2}\right)=0.25<0.35=\lambda_{\mathbf{2}} \Rightarrow \operatorname{not}\left(a S_{\mathbf{2}} b_{2}\right)$, and $C_{\mathbf{2}}\left(b_{2}, a\right)=0.5 \geq 0.35=\lambda_{\mathbf{2}} \Rightarrow b_{2} S_{\mathbf{2}} a$.

For $\gamma$ ), $a ?_{\mathbf{2}} b_{1}$, while for $\left.\delta\right), b_{2} \succ_{\mathbf{2}} a$. Consequently, the optimistic procedure applied in $G_{\mathbf{2}}$ will make the following assignment: $a \underset{\mathbf{2}}{\rightarrow} C l_{2}$.

Looking, in turn, at Table 1(d), we can observe that

e) $C_{\mathbf{0}}\left(a, b_{1}\right)=0.65<0.7=\lambda_{\mathbf{0}} \Rightarrow \operatorname{not}\left(a S_{\mathbf{0}} b_{1}\right)$, and $C_{\mathbf{0}}\left(b_{1}, a\right)=0.75 \geq 0.7=\lambda_{\mathbf{0}} \Rightarrow b_{1} S_{\mathbf{0}} a$.

For $\theta), b_{1} \succ_{\mathbf{0}} a$ and, consequently, the optimistic procedure applied in $G_{\mathbf{0}}$ will make the following assignment: $a \underset{\mathbf{0}}{\rightarrow} C l_{1}$.

The above example concerns the problem of sorting, however, an analogical situation may also happen when alternatives are compared pairwise, like in the choice or ranking problem in the presence of hierarchical criteria, and subject to interpretation of both outranking and inverse outranking relation on a pair $a, b$ 
that gives preference, or indifference, or incomparability. Indeed, even if alternative $a$ is not preferred to alternative $b$ with respect to any of the subcriteria $G_{(\mathbf{r}, 1)}, \ldots, G_{(\mathbf{r}, n(\mathbf{r}))}$ of criterion $G_{\mathbf{r}}$, when $a$ is indifferent to $b$ on some of these subcriteria and $a$ is incomparable with $b$ on the remaining subcriteria, it is still possible that $a$ is preferred to $b$ with respect to criterion $G_{\mathbf{r}}$. We stressed that $a$ is indifferent to $b$ on some subcriteria and it is incomparable with $b$ on the remaining ones since, for the coherence properties C1) and C2), if $a$ is indifferent to $b$ with respect to all $G_{(\mathbf{r}, j)}, j=1, \ldots, n(\mathbf{r})$, then $a$ is indifferent to $b$ also with respect to $G_{\mathbf{r}}{ }^{3}$; analogously, if $a$ is incomparable with $b$ with respect to all subcriteria $G_{(\mathbf{r}, j)}, j=1, \ldots, n(\mathbf{r})$, then $a$ is incomparable with $b$ also with respect to $G_{\mathbf{r}}{ }^{4}$ We deferred to the Appendix B, the formal explanation of the reason for which the strange situation highlighted in Note 4.1 can occur.

The reader could argue that more than being strange, the previous situation should not occur in practice since it may seem inadmissible that an alternative $a$ is assigned on criterion $G_{\mathbf{r}}$ to a category lower than the minimum category to which the same alternative is assigned on the subcriteria directly descending from $G_{\mathbf{r}}$. For this reason, we propose a new procedure that avoids this strange situation. The proposed modification of the optimistic procedure is composed of the following steps:

step 1) compute the optimistic assignment of alternative $a$ with respect to subcriteria $G_{(\mathbf{r}, 1)}, \ldots, G_{(\mathbf{r}, n(\mathbf{r}))}$ and denote by $C l_{k_{1}}, \ldots, C l_{k_{n(\mathbf{r})}}$ the categories to which alternative $a$ is assigned with respect to these subcriteria,

step 2) compute the optimistic assignment of alternative $a$ with respect to subcriterion $G_{\mathbf{r}}$ and denote by $C l_{k}$ the category to which $a$ is assigned with respect to this subcriterion,

step 3) assign alternative $a$ with respect to criterion $G_{\mathbf{r}}$ to category $C l_{k^{\prime}}$ where $k^{\prime}=\max \left\{k, \min \left\{k_{1}, \ldots, k_{n(\mathbf{r})}\right\}\right\}$.

By using the proposed modification of the optimistic procedure, the category $C l_{k^{\prime}}$ to which $a$ is assigned with respect to criterion $G_{\mathbf{r}}$ is for sure not lower than the minimum category to which $a$ is assigned with respect to subcriteria $G_{(\mathbf{r}, 1)}, \ldots, G_{(\mathbf{r}, n(\mathbf{r}))}$. In particular, by using the proposed procedure, in the previous example, alternative $a$ should be assigned to category $\mathrm{Cl}_{2}$ with respect to criterion $G_{\mathbf{0}}$. Indeed, since according to step 2), $a$ is assigned to category $C l_{2}$, both with respect to subcriteria $G_{\mathbf{1}}$ and $G_{\mathbf{2}}$ (therefore $k_{1}=k_{2}=2$ ) and it is assigned to category $C l_{1}$ with respect to criterion $G_{\mathbf{0}}(k=1)$, then according to step $\left.\mathbf{3}\right)$, the new category to which $a$ should be assigned with respect to criterion $G_{0}$ is $C l_{2}$ :

$$
k^{\prime}=\max \left\{k, \min \left\{k_{1}, k_{2}\right\}\right\}=\max \{1, \min \{2,2\}\}=\max \{1,2\}=2 .
$$

\footnotetext{
${ }^{3}$ If $a$ is indifferent to $b$ with respect to all $G_{(\mathbf{r}, j)}$, then $a S_{(\mathbf{r}, j)} b$ and $b S_{(\mathbf{r}, j)} a$ for all $j=1, \ldots, n(\mathbf{r})$. Consequently, by coherence property $\mathbf{C 1}$ ), $a S_{\mathbf{r}} b$ and $b S_{\mathbf{r}} a$ and, therefore, $a \sim_{\mathbf{r}} b$

${ }^{4}$ If $a$ is incomparable with $b$ with respect to all $G_{(\mathbf{r}, j)}$, then $\operatorname{not}\left(a S_{(\mathbf{r}, j)} b\right)$ and $\operatorname{not}\left(b S_{(\mathbf{r}, j)} a\right)$ for all $j=1, \ldots, n(\mathbf{r})$. Consequently, by coherence property $\mathbf{C 2}), \operatorname{not}\left(a S_{\mathbf{r}} b\right)$ and $\operatorname{not}\left(b S_{\mathbf{r}} a\right)$ and, therefore, $a ? \mathbf{r} b$
} 


\subsection{ELECTRE-Tri $C$ and $M C H P$}

In contrast to ELECTRE Tri-B, where each category $C l_{h}$ is characterized by two reference profiles corresponding to the limits of this category, in ELECTRE Tri-C each category $C l_{h}$ is characterized by one reference profile $b_{h}$ only, being representative of the category. The construction of the credibility indices is the same as that of ELECTRE Tri-B. Considering $p$ categories, in addition to the reference profiles $b_{1}, \ldots, b_{p}$ belonging to set $B$ and being representative of the categories $C l_{1}, \ldots, C l_{p}$, two other extreme profiles, $b_{0}$ and $b_{p+1}$, have to be defined, such that $g_{\mathbf{t}}\left(b_{0}\right)<g_{\mathbf{t}}(a)<g_{\mathbf{t}}\left(b_{p+1}\right), g_{\mathbf{t}}\left(b_{1}\right)>g_{\mathbf{t}}\left(b_{0}\right)$ and $g_{\mathbf{t}}\left(b_{p+1}\right)>g_{\mathbf{t}}\left(b_{p}\right)$ for all $\mathbf{t} \in E L$. In order to adapt ELECTRE Tri-C to MCHP, following [2], two reference profiles, $b_{h+1}$ and $b_{h}$, define two distinct categories only if $b_{h+1}$ is at least weakly preferred to $b_{h}$ on at least one elementary criterion (i.e., $b_{h+1} Q_{\mathbf{t}} b_{h}$ for some $\left.\mathbf{t} \in E L\right)$. For this reason, we shall impose that $\sigma_{\mathbf{s}}\left(b_{h}, b_{h+1}\right)<W_{s}$ for all $\mathbf{s} \in L B O$ and for all $h=0, \ldots, p$.

Remark 4.1. Let us observe that imposing $\sigma_{\mathbf{s}}\left(b_{h}, b_{h+1}\right)<W_{\mathbf{s}}$ for all $\mathbf{s} \in L B O$ and for all $h=0, \ldots, p$, we get that $\sigma_{0}\left(b_{h}, b_{h+1}\right)<1$ for all $h=0, \ldots, p$, being the weak separability condition in ELECTRE Tri-C.

More distinct categories can be obtained by imposing the strict and the hyper-strict separability conditions. Regarding the application of MCHP to ELECTRE Tri-C, the strict and hyper-strict separability conditions defined for non-hierarchical ELECTRE Tri-C can be redefined imposing that $\sigma_{\mathbf{s}}\left(b_{h}, b_{h+1}\right)<\frac{W_{\mathbf{s}}}{2}$ and $\sigma_{\mathbf{s}}\left(b_{h}, b_{h+1}\right)=0$ for all $\mathbf{s} \in L B O$ and for all $h=0, \ldots, p$, respectively. On one hand, the strict separability condition imposes that less than a half of elementary criteria descending from criterion $G_{\mathbf{s}}, \mathbf{s} \in L B O$, have to be in favor of the outranking of $b_{h}$ on $b_{h+1}$ with respect to criterion $G_{\mathbf{s}}$, for all $h=0, \ldots, p$. On the other hand, imposing the hyper-strict separability condition is equivalent to impose that $b_{h+1}$ is strictly preferred to $b_{h}$ for all elementary criteria.

When MCHP is applied to ELECTRE Tri-C, for each criterion $G_{\mathbf{r}}, \mathbf{r} \in \mathcal{I}_{\mathcal{G}} \backslash E L$, we can define the two following assignment rules:

- Descending rule: For a chosen cutting level $\lambda_{\mathbf{r}} \in\left[W_{\mathbf{r}} / 2, W_{\mathbf{r}}\right]$, decrease $h$ from $p$ until the first value $l$, such that $\sigma_{\mathbf{r}}\left(a, b_{l}\right) \geq \lambda_{\mathbf{r}}$ :

- for $l=p$, assign $a$ to category $C l_{p}$,

- for $0<l<p$, if $\rho_{\mathbf{r}}\left(a, b_{l}\right)>\rho_{\mathbf{r}}\left(a, b_{l+1}\right)$, then assign $a$ to category $C l_{l}$, otherwise assign $a$ to $C l_{l+1}$,

- for $l=0$, assign $a$ to category $C l_{1}$.

- Ascending rule: For a chosen cutting level $\lambda_{\mathbf{r}} \in\left[W_{\mathbf{r}} / 2, W_{\mathbf{r}}\right]$, increase $h$ from one until the first value $l$, such that $\sigma_{\mathbf{r}}\left(b_{l}, a\right) \geq \lambda_{\mathbf{r}}$ :

- for $l=1$, assign $a$ to category $C l_{1}$, 
- for $1<l<p+1$, if $\rho_{\mathbf{r}}\left(a, b_{l}\right)>\rho_{\mathbf{r}}\left(a, b_{l-1}\right)$, then assign $a$ to category $C l_{l}$, otherwise assign $a$ to $C l_{l-1}$,

- for $k=p+1$, assign $a$ to category $C l_{p}$.

In both the descending and the ascending rules, $\rho_{\mathbf{r}}\left(a, b_{h}\right)=\min \left\{\sigma_{\mathbf{r}}\left(a, b_{h}\right), \sigma_{\mathbf{r}}\left(b_{h}, a\right)\right\}$.

\subsection{ELECTRE Tri-nC and MCHP}

In contrast to ELECTRE Tri-C, where each category $C l_{h}$ is characterized by one reference profile only, in ELECTRE Tri-nC, each category $C l_{h}$ is represented by $m_{h} \geq 1$ reference profiles. Denoting by $B_{h}=$ $\left\{b_{h}^{1}, \ldots, b_{h}^{m_{h}}\right\}$ the set of reference profiles defining category $C l_{h}$, in order to apply MCHP to ELECTRE Tri-nC, we have to consider $p+2$ sets $B_{0}, B_{1}, \ldots, B_{p}, B_{p+1}$, where $B_{0}$ and $B_{p+1}$ are composed, respectively, of the extreme reference profiles $b_{0}^{1}$ and $b_{p+1}^{1}$, such that $g_{\mathbf{t}}\left(b_{0}^{1}\right)<g_{\mathbf{t}}(a)<g_{\mathbf{t}}\left(b_{p+1}^{1}\right), g_{\mathbf{t}}\left(b_{1}^{k}\right)>g_{\mathbf{t}}\left(b_{0}^{1}\right)$ and $g_{\mathbf{t}}\left(b_{p+1}^{1}\right)>g_{\mathbf{t}}\left(b_{p}^{l}\right)$ for all $k=1, \ldots, m_{1}, l=1, \ldots, m_{p}$, and for all $\mathbf{t} \in E L$.

Because each category $C l_{h}$ is now represented by a set of reference profiles $B_{h}$, the separability conditions given in ELECTRE Tri-C are reformulated in ELECTRE Tri-nC as follows:

- weak separability condition: $\sigma_{\mathbf{s}}\left(b_{h}^{k}, b_{h+1}^{l}\right)<W_{\mathbf{s}}$, for all $\mathbf{s} \in L B O, k=1, \ldots, m_{h}, l=1, \ldots, m_{h+1}$, and $h=1, \ldots, p-1$,

- strict separability condition: $\sigma_{\mathbf{s}}\left(b_{h}^{k}, b_{h+1}^{l}\right)<\frac{W_{\mathbf{s}}}{2}$, for all $\mathbf{s} \in L B O, k=1, \ldots, m_{h}, l=1, \ldots, m_{h+1}$, and $h=1, \ldots, p-1$,

- hyper-strict separability condition: $\sigma_{\mathbf{s}}\left(b_{h}^{k}, b_{h+1}^{l}\right)=0$, for all $\mathbf{s} \in L B O, k=1, \ldots, m_{h}, l=1, \ldots, m_{h+1}$, and $h=1, \ldots, p-1$.

Note 4.2. We note again that imposing the separability conditions for all $\mathbf{s} \in L B O$, we get the separability conditions on the whole hierarchy of criteria, as in ELECTRE Tri-nC.

The assignment procedure for an alternative $a \in A$ with respect to criterion $G_{\mathbf{r}}, \mathbf{r} \in \mathcal{I}_{G}$, is based on the computation of the credibility indices $\sigma_{\mathbf{r}}\left(\{a\}, B_{h}\right)$ and $\sigma_{\mathbf{r}}\left(B_{h},\{a\}\right)$ defined for all $h=1, \ldots, p$ as follows:

- $\sigma_{\mathbf{r}}\left(\{a\}, B_{h}\right)=\max _{k=1, \ldots, m_{h}}\left\{\sigma_{\mathbf{r}}\left(a, b_{h}^{k}\right)\right\}$,

- $\sigma_{\mathbf{r}}\left(B_{h},\{a\}\right)=\max _{k=1, \ldots, m_{h}}\left\{\sigma_{\mathbf{r}}\left(b_{h}^{k}, a\right)\right\}$.

Given a criterion $G_{\mathbf{r}}, \mathbf{r} \in \mathcal{I}_{\mathcal{G}} \backslash E L$, two assignment rules can be defined for ELECTRE Tri-nC:

- Descending rule: For a chosen cutting level $\lambda_{\mathbf{r}}$, decrease $h$ from $p$ until the first value $l$, such that $\sigma_{\mathbf{r}}\left(\{a\}, B_{l}\right) \geq \lambda_{\mathbf{r}}:$

- for $l=p$, assign $a$ to category $C l_{p}$, 
- for $0<l<p$, if $\rho_{\mathbf{r}}\left(\{a\}, B_{l}\right)>\rho_{\mathbf{r}}\left(\{a\}, B_{l+1}\right)$, then assign $a$ to category $C l_{l}$, otherwise assign $a$ to $C l_{l+1}$,

- for $l=0$, assign $a$ to category $C l_{1}$.

- Ascending rule: For a chosen cutting level $\lambda_{\mathbf{r}}$, increase $h$ from one until the first value $l$, such that $\sigma_{\mathbf{r}}\left(B_{l},\{a\}\right) \geq \lambda_{\mathbf{r}}:$

- for $l=1$, assign $a$ to category $C l_{1}$,

- for $1<l<p+1$, if $\rho_{\mathbf{r}}\left(\{a\}, B_{l}\right)>\rho_{\mathbf{r}}\left(\{a\}, B_{l-1}\right)$, then assign $a$ to category $C l_{l}$, otherwise assign $a$ to $C l_{l-1}$

- for $l=p+1$, assign $a$ to category $C l_{p}$.

In both the descending and the ascending rules, we define $\rho_{\mathbf{r}}\left(\{a\}, B_{h}\right)=\min \left\{\sigma_{\mathbf{r}}\left(\{a\}, B_{h}\right), \sigma_{\mathbf{r}}\left(B_{h},\{a\}\right)\right\}$.

Note 4.3. Let us note that the pessimistic and optimistic assignments of ELECTRE Tri-B, as well as the ascending and descending rules of ELECTRE Tri-C and ELECTRE Tri-nC for a flat structure of criteria, can be easily obtained by considering $G_{\mathbf{r}}=G_{\mathbf{0}}$, that is by considering the entire set of criteria and not a particular criterion or subcriterion only. Indeed, the rules defined for the extensions of ELECTRE Tri methods to the case of hierarchy of criteria are exactly the same as the rules defined for ELECTRE Tri methods considering a flat structure of criteria, excepting that in these rules we consider a particular criterion in the hierarchy only. Moreover, $C_{\mathbf{0}}(a, b)=C(a, b)$ for all $a, b \in A$, where $C(a, b)$ is the comprehensive concordance index of the classical ELECTRE methods.

\subsection{Determining the weights of criteria in hierarchical ELECTRE Tri methods}

A typical approach to determine the weights of criteria in the ELECTRE methods is the Simos-RoyFigueira (SRF) method that has been proposed by Figueira and Roy [14], improving the original proposal of Simos [19, 20]. Using the SRF method, each criterion is written on one card, and there are also empty cards. The DM is asked to put the cards in a rank order from the least important to the most important. Criteria that are considered as equally important are getting the same rank. Moreover, the DM can put one or more empty cards separating some criteria in the rank order. Each empty card is put in one rank. The greater the number of empty cards separating two sets of indifferent criteria, the greater is the difference of importance between these sets of criteria. Thus, if $\mathcal{J}=\{1, \ldots, m\}$ is the set of considered criteria, let us denote by $L_{1}$ the set of the least important criteria, by $L_{2}$ the set of second least important criteria, and so on, until $L_{v}$ being the set of the most important criteria, $L_{1}, L_{2}, \ldots, L_{v} \subseteq \mathcal{J}, L_{s} \cap L_{t}=\emptyset$ for all $s \neq t, s, t=1, \ldots, v$. Let us denote by $e_{s}$ the number of empty cards between $L_{s}$ and $L_{s+1}, s=1, \ldots, v-1$. Moreover, the DM is asked to define a ratio $z$ between the weights of criteria from $L_{1}$ and criteria from $L_{v}$. 
Using this information, to each criterion $j \in \mathcal{J}$ is assigned the following non-normalized weight:

$$
w_{j}^{\prime}=1+\frac{(z-1)\left[l(j)-1+\sum_{s=1}^{l(j)-1} e_{s}\right]}{v-1+\sum_{s=1}^{v-1} e_{s}}
$$

where $l(j)$ denotes the rank of importance to which criterion $j$ belongs, i.e., $j \in L_{l(j)}$. The normalized weight $w_{j}$ assigned to each criterion $j \in \mathcal{J}$ is therefore obtained as follows:

$$
w_{j}=\frac{w_{j}^{\prime}}{\sum_{i \in \mathcal{J}} w_{i}^{\prime}}
$$

The same procedure can be used in case of the hierarchy of criteria as follows. Starting from the root $G_{\mathbf{0}}$, and continuing from the top to the bottom, for each criterion $G_{\mathbf{r}}, \mathbf{r} \in \mathcal{I}_{\mathcal{G}} \backslash E L$, the DM is asked to rank the criteria $G_{(\mathbf{r}, 1)}, \ldots, G_{(\mathbf{r}, n(\mathbf{r}))}$ from the least important to the most important, with $L_{(\mathbf{r}, 1)}$ the set of the least important criteria and $L_{(\mathbf{r}, v(\mathbf{r}))}$ the set of the most important criteria, such that $L_{\left(\mathbf{r}, s_{1}\right)} \cap L_{\left(\mathbf{r}, s_{2}\right)}=\emptyset$ for all $s_{1} \neq s_{2}$, and $s_{1}, s_{2}=1, \ldots, v(\mathbf{r})$. Let us denote by $e_{(\mathbf{r}, s)}$ the number of empty cards between sets $L_{(\mathbf{r}, s)}$ and $L_{(\mathbf{r}, s+1)}, s=1, \ldots, v(\mathbf{r})-1$. Moreover, the DM is asked to define a ratio $z_{\mathbf{r}}$ between the weight of criteria from $L_{(\mathbf{r}, 1)}$, and criteria from $L_{(\mathbf{r}, v(\mathbf{r}))}$. Using the SRF method, a locally normalized weight $w_{(\mathbf{r}, j)}^{*}$ is assigned to each criterion $G_{(\mathbf{r}, j)}, j=1, \ldots, n(\mathbf{r})$, as follows:

$$
w_{(\mathbf{r}, j)}^{*}=\frac{w_{(\mathbf{r}, j)}^{\prime}}{\sum_{s=1}^{n(\mathbf{r})} w_{(\mathbf{r}, s)}^{\prime}}
$$

where

$$
w_{(\mathbf{r}, j)}^{\prime}=1+\frac{\left(z_{\mathbf{r}}-1\right)\left[l(\mathbf{r}, j)-1+\sum_{s=1}^{l(\mathbf{r}, j)-1} e_{(\mathbf{r}, s)}\right]}{v(\mathbf{r})-1+\sum_{s=1}^{v(\mathbf{r})-1} e_{(\mathbf{r}, s)}}
$$

and $l(\mathbf{r}, s)$ denotes the rank to which criterion $G_{(\mathbf{r}, s)}$ belongs, i.e., $(\mathbf{r}, s) \in L_{l(\mathbf{r}, s)}$. The final globally normalized weights are, therefore, obtained as follows:

- $w_{\mathbf{r}}=w_{\mathbf{r}}^{*}$ for all $G_{\mathbf{r}}$ in the first level of the hierarchy,

- $w_{(\mathbf{r}, s)}=w_{\mathbf{r}} w_{(\mathbf{r}, s)}^{*}, s=1, \ldots, n(\mathbf{r})$, where $w_{\mathbf{r}}$ is the globally normalized weight of criterion $G_{\mathbf{r}}$.

This means that the weight $w_{\mathbf{r}}$ assigned to the first level criterion $G_{\mathbf{r}}$ is that one obtained by applying the SRF method to the set composed of all first level criteria, while the weight $w_{(\mathbf{r}, s)}$ assigned to any criterion 
$G_{(\mathbf{r}, s)}$ in the lower level of the hierarchy is obtained by multiplying the weight $w_{(\mathbf{r}, s)}^{*}$ of $G_{(\mathbf{r}, s)}$, obtained by the SRF method for the set of criteria $\left\{G_{(\mathbf{r}, s)}, s=1, \ldots, n(\mathbf{r})\right\}$, by the weight of criterion $G_{\mathbf{r}}$ from the higher level of the hierarchy $\left(G_{\mathbf{r}}\right.$ being the direct parent of $\left.G_{(\mathbf{r}, s)}\right)$.

Observe that

- $\sum_{s=1}^{n(\mathbf{r})} w_{(\mathbf{r}, s)}^{*}=1$ for all criteria $G_{\mathbf{r}}, \mathbf{r} \in \mathcal{I}_{\mathcal{G}} \backslash E L$,

- $\sum_{\mathbf{t} \in E L} w_{\mathbf{t}}=1$.

Figure 2: Example of the application of the SRF method for hierarchical criteria

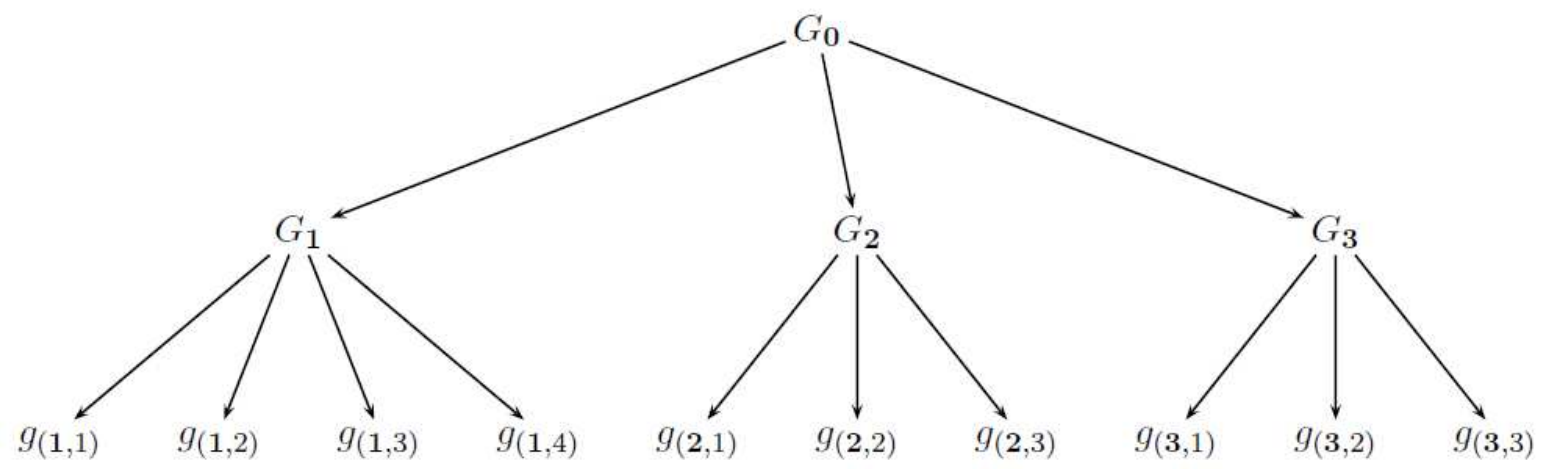

The following example illustrates the application of the SRF method to a hierarchy of criteria. Let us suppose we have a set of criteria organized in two different levels, as shown in Fig. 2.

Suppose that the DM orders first the first level criteria, and then the second level (elementary) criteria. The ordering always starts from the least important to the most important criterion in the considered set. In our example, the DM put the first level criteria in the following order: $G_{\mathbf{1}}, G_{\mathbf{3}}, G_{\mathbf{2}}$. Moreover, he put one empty card between $G_{\mathbf{1}}$ and $G_{\mathbf{3}}$, and two empty cards between $G_{\mathbf{3}}$ and $G_{\mathbf{2}}$. For him, $G_{\mathbf{2}}$ is ten times more important than $G_{\mathbf{1}}$, so $z=10$. By applying the SRF method to this set of criteria we get the following weights: $w_{\mathbf{1}}=w_{\mathbf{1}}^{*}=0.064, w_{\mathbf{2}}=w_{\mathbf{2}}^{*}=0.641$ and $w_{\mathbf{3}}=w_{\mathbf{3}}^{*}=0.294$. After that, the DM gave the following order of subcriteria from $G_{\mathbf{1}}: g_{(\mathbf{1}, 3)}, g_{(\mathbf{1}, 2)}$, and in the same rank $g_{(\mathbf{1}, 1)}$ and $g_{(\mathbf{1}, 4)}$. He also put one empty card between $g_{(\mathbf{1}, 3)}$ and $g_{(\mathbf{1}, 2)}$, and stated that the first rank criteria $\left(g_{(\mathbf{1}, 1)}\right.$ and $\left.g_{(\mathbf{1}, 4)}\right)$ are three times more important than the last rank criterion $\left(g_{(\mathbf{1}, 3)}\right)$, so that $z_{\mathbf{1}}=3$. Applying the SRF method to these four criteria we get the weights $w_{(\mathbf{1}, 1)}^{*}=0.321, w_{(\mathbf{1}, 2)}^{*}=0.25, w_{(\mathbf{1}, 3)}^{*}=0.107, w_{(\mathbf{1}, 4)}^{*}=0.321$. Since these criteria are subcriteria of $G_{\mathbf{1}}$ whose weight is $w_{\mathbf{1}}=0.064$, the weights $w_{(\mathbf{1}, i)}^{*}, i=1, \ldots, 4$, should be multiplied by $w_{\mathbf{1}}$. Consequently, we get the final weights $w_{(\mathbf{1}, 1)}=0.02, w_{(\mathbf{1}, 2)}=0.016, w_{(\mathbf{1}, 3)}=0.006, w_{(\mathbf{1}, 4)}=0.02$. Proceeding analogously for the two other subsets of elementary criteria, one gets the weights presented in Table 2.

Let us observe that the proposed extension of the SRF method can be applied not only to ELECTRE methods dealing with sorting decision problems but also to all other hierarchical MCDA methods where it 
Table 2: Example of the application of the SRF method for hierarchical criteria. The criteria are ordered in the order of an increasing importance. The higher the rank, the higher the importance of criteria in that rank.

(a) First level criteria with $z=10$.

(b) Subcriteria of first level criterion $G_{1}$ with $z_{1}=3$.

\begin{tabular}{|cccc|}
\hline Rank & Criteria & $e_{r}$ & $w_{s}=w_{s}^{*}$ \\
\hline 1 & $G_{\mathbf{1}}$ & 1 & 0.0641 \\
2 & $G_{\mathbf{3}}$ & 2 & 0.2948 \\
3 & $G_{\mathbf{2}}$ & & 0.6410 \\
\hline
\end{tabular}

\begin{tabular}{|ccccc|}
\hline Rank & Criteria & $e_{(\mathbf{1}, s)}$ & $w_{(\mathbf{1}, s)}^{*}$ & $w_{(\mathbf{1}, s)}=w_{(\mathbf{1}, s)}^{*} \times w_{\mathbf{1}}$ \\
\hline 1 & $g_{(\mathbf{1}, 3)}$ & 1 & 0.1071 & 0.0068 \\
2 & $g_{(\mathbf{1}, 2)}$ & 0 & 0.2500 & 0.0160 \\
3 & $\left\{g_{(\mathbf{1}, 1)}, g_{(\mathbf{1}, 4)}\right\}$ & & 0.3214 & 0.0206 \\
\hline
\end{tabular}

(c) Subcriteria of first level criterion $G_{2}$ with $z_{2}=5$.

\begin{tabular}{|ccccc|}
\hline Rank & Criteria & $e_{(\mathbf{2}, s)}$ & $w_{(\mathbf{2}, s)}^{*}$ & $w_{(\mathbf{2}, s)}=w_{(\mathbf{2}, s)}^{*} \times w_{\mathbf{2}}$ \\
\hline 1 & $\left\{g_{(\mathbf{2}, 2)}, g_{(\mathbf{2}, 3)}\right\}$ & 1 & 0.1428 & 0.0421 \\
2 & $g_{(\mathbf{2}, 1)}$ & 0 & 0.7142 & 0.2106 \\
\hline
\end{tabular}

(d) Subcriteria of first level criterion $G_{\mathbf{3}}$ with $z_{\mathbf{3}}=7$.

\begin{tabular}{|ccccc|}
\hline Rank & Criteria & $e_{(\mathbf{3}, s)}$ & $w_{(\mathbf{3}, s)}^{*}$ & $w_{(\mathbf{3}, s)}=w_{(\mathbf{3}, s)}^{*} \times w_{\mathbf{3}}$ \\
\hline 1 & $g_{(\mathbf{3}, 3)}$ & 2 & 0.0740 & 0.0474 \\
2 & $g_{(\mathbf{3}, 1)}$ & 0 & 0.4074 & 0.2611 \\
3 & $g_{(\mathbf{3}, 2)}$ & & 0.5185 & 0.3323 \\
\hline
\end{tabular}

is meaningful to use criteria weights having the interpretation of relative voting power in non-compensatory aggregation, and not of substitution rates like in the weighted sum.

\subsection{ELECTRE Tri with interactions between criteria}

In the classical ELECTRE methods, it is supposed that there is not any significant interaction between criteria but, in many real world decision problems, the evaluation criteria could present a certain degree of interaction that, according to [13], can be of three types: mutual strengthening effect (synergy), mutual weakening effect (redundancy), and antagonistic effect. For example, when evaluating sport cars, the criteria maximum speed and acceleration may be considered redundant because, in general, speedy cars have also a good acceleration. Therefore, even if these two criteria can be very important for a DM liking sport cars, their comprehensive importance is smaller than the sum of the importance of the two criteria considered separately. On the other hand, the criteria maximum speed and price contribute to a synergy effect because a speedy car with a low price is very well appreciated. For this reason, the comprehensive importance of these two criteria should be greater than the sum of importance of the two criteria considered separately. Besides, when evaluating a car having good price but bad safety, the weight of criterion price can be reduced because even if the DM appreciates the low price she is really concerned about the safety of the car. In the latter case, the two criteria are antagonistic.

In this section, we shall describe how to deal with the interaction between any two criteria in case of the MCHP applied to ELECTRE Tri methods.

Considering the elementary criterion $g_{\mathbf{t}_{1}}$ in favour of the outranking of an alternative $a$ over an alternative $b$, we have that:

- if elementary criterion $g_{\mathbf{t}_{2}}$ is in favour of the outranking of $a$ over $b$ and both criteria present a certain 
degree of synergy, then this synergy is considered by adding a value $w_{\mathbf{t}_{1} \mathbf{t}_{2}}>0$ in the computation of the concordance index $C(a, b)$,

- if elementary criterion $g_{\mathbf{t}_{2}}$ is in favour of the outranking of $a$ over $b$ but both criteria present a certain degree of redundancy, then this redundancy is considered by adding a value $w_{\mathbf{t}_{1} \mathbf{t}_{2}}<0$ in the computation of the concordance index $C(a, b)$,

- if elementary criterion $g_{\mathbf{t}_{2}}$ is opposed to the outranking and the DM decides that there is an antagonism between the two criteria, then the antagonistic effect is considered by adding a value $w_{\mathbf{t}_{1} \mathbf{t}_{2}}^{\prime}<0$ in the computation of the concordance index $C(a, b)$.

Let us observe that in case of synergy or redundancy of two elementary criteria, we have $w_{\mathbf{t}_{1} \mathbf{t}_{2}}=w_{\mathbf{t}_{2} \mathbf{t}_{1}}$, while in case of antagonism between two elementary criteria it is possible that $w_{\mathbf{t}_{1} \mathbf{t}_{2}}^{\prime} \neq w_{\mathbf{t}_{2} \mathbf{t}_{1}}^{\prime}$.

In [13], in order to avoid that interactions between criteria can render negative the contribution of each criterion, a positive net balance has been considered. It can be expressed as follows:

Condition 4.1. For all $\mathbf{t}_{1} \in E L$,

$$
w_{\mathbf{t}_{1}}-\left(\sum_{\mathbf{t}_{2} \in E L: w_{\mathbf{t}_{1} \mathbf{t}_{2}}<0}\left|w_{\mathbf{t}_{1} \mathbf{t}_{2}}\right|+\sum_{\mathbf{t}_{3} \in E L} w_{\mathbf{t}_{1} \mathbf{t}_{3}}^{\prime}\right)>0
$$

Moreover, in the MCHP case, we need that this condition holds not only comprehensively but also at each node of the hierarchy. As a consequence, Condition 4.1 has to be extended as follows:

Condition 4.2. For all $G_{\mathbf{r}}, \mathbf{r} \in \mathcal{I}_{\mathcal{G}} \backslash E L$, and for all $\mathbf{t}_{1} \in E\left(G_{\mathbf{r}}\right)$,

$$
w_{\mathbf{t}_{1}}-\left(\sum_{\mathbf{t}_{2} \in E\left(G_{\mathbf{r}}\right): w_{\mathbf{t}_{1} \mathbf{t}_{2}<0}}\left|w_{\mathbf{t}_{1} \mathbf{t}_{2}}\right|+\sum_{\mathbf{t}_{3} \in E\left(G_{\mathbf{r}}\right)} w_{\mathbf{t}_{1} \mathbf{t}_{3}}^{\prime}\right)>0
$$

Let us observe that (10) can be seen as a particular case of (11) if we consider $G_{\mathbf{r}}=G_{\mathbf{0}}$. Note, moreover, that Condition 4.2 has to be satisfied when hierarchy and interaction of criteria are considered simultaneously in any type of decision problem, i.e., not only sorting but also choice and ranking.

Using the notation introduced in Section 2 , for each criterion $G_{\mathbf{r}}, \mathbf{r} \in \mathcal{I}_{\mathcal{G}}$, and for each $(a, b)$, we can define the following sets:

- $L_{\mathbf{r}}(a, b)$ being the set of all couples $\left\{\mathbf{t}_{1}, \mathbf{t}_{2}\right\} \subseteq E\left(G_{\mathbf{r}}\right)$, such that $\mathbf{t}_{1}, \mathbf{t}_{2} \in \bar{C}(b P a)$,

- $O_{\mathbf{r}}(a, b)$ being the set of all pairs $\left(\mathbf{t}_{1}, \mathbf{t}_{2}\right) \in E\left(G_{\mathbf{t}}\right) \times E\left(G_{\mathbf{t}}\right)$, such that $\mathbf{t}_{1} \in \bar{C}(b P a)$ and $\mathbf{t}_{2} \in C(b P a)$.

$C(b T a)$ is the coalition of elementary criteria in favor of the assertion $b T a$, where $T \in\{S, Q, P\}$ (see equation $(1)), C(b P a)=C(a S b) \cup C(b Q a)$, and $\bar{C}(b P a)$ denotes the complement of $C(b P a)$. 
Now, we can define the comprehensive and partial concordance indices in MCHP taking into account interactions between criteria.

Definition 4.2. For each pair of alternatives $(a, b) \in A \times A$, and for each criterion $G_{\mathbf{r}}, \mathbf{r} \in \mathcal{I}_{\mathcal{G}}$,

$$
\begin{aligned}
C_{\mathbf{r}}(a, b)=\frac{1}{W_{\mathbf{r}}(a, b)}\left(\sum_{\mathbf{t}_{1} \in \bar{C}(b P a) \cap E\left(G_{\mathbf{r}}\right)} w_{\mathbf{t}_{1}} \phi_{\mathbf{t}_{1}}(a, b)+\sum_{\left\{\mathbf{t}_{1}, \mathbf{t}_{2}\right\} \in L_{\mathbf{r}}(a, b)} w_{\mathbf{t}_{1} \mathbf{t}_{2}} Z\left(\phi_{\mathbf{t}_{1}}(a, b), \phi_{\mathbf{t}_{2}}(a, b)\right)-\right. \\
\\
\left.\quad-\sum_{\left(\mathbf{t}_{1}, \mathbf{t}_{2}\right) \in O_{\mathbf{r}}(a, b)} w_{\mathbf{t}_{1} \mathbf{t}_{2}}^{\prime} Z\left(\phi_{\mathbf{t}_{1}}(a, b), \phi_{\mathbf{t}_{2}}(b, a)\right)\right)
\end{aligned}
$$

where

$$
W_{\mathbf{r}}(a, b)=\sum_{\mathbf{t} \in E\left(G_{\mathbf{r}}\right)} w_{\mathbf{t}}+\sum_{\left\{\mathbf{t}_{1}, \mathbf{t}_{2}\right\} \in L_{\mathbf{r}}(a, b)} w_{\mathbf{t}_{1} \mathbf{t}_{2}} Z\left(\phi_{\mathbf{t}_{1}}(a, b), \phi_{\mathbf{t}_{2}}(a, b)\right)-\sum_{\left(\mathbf{t}_{1}, \mathbf{t}_{2}\right) \in O_{\mathbf{r}}(a, b)} w_{\mathbf{t}_{1} \mathbf{t}_{2}}^{\prime} Z\left(\phi_{\mathbf{t}_{1}}(a, b), \phi_{\mathbf{t}_{2}}(b, a)\right)
$$

and $Z:[0,1]^{2} \rightarrow[0,1]$ is a function non-decreasing in both its two arguments, such that $Z(1, x)=Z(x, 1)=x$ for all $x \in[0,1]$. For example, we can have $Z(x, y)=x y$ or $Z(x, y)=\min (x, y)$.

In order to take into account the possible interactions between criteria in the application of MCHP to ELECTRE Tri methods, it is sufficient to replace the comprehensive and partial concordance indices by the one given in Definition 4.2.

\section{Didactic Example}

In this section, we shall present the application of the hierarchical ELECTRE Tri-B method to a real world decision problem. In the year 2000, the Transport Infrastructure Regional Study (TIRS) has been undertaken in the context of the stability pact under the lead of the European Investment Bank (EIB) and European Union (EU) to evaluate the feasibility of the realization of 223 projects regarding roads, ports and airports in the Balkans area and, in particular, in Albania, Bosnia and Herzegovina, Bulgaria, Croatia, Serbia, Macedonia and Romania. The different projects have been evaluated with respect to 11 elementary criteria (see [1] for further details on TIRS). Taking into account the nature of the elementary criteria, one can build the hierarchy of criteria shown in Figure 3. Two macro-criteria are considered at the first level of the hierarchy tree:

- the socio-economic return on investment $\left(G_{\mathbf{1}}\right)$,

- the functionality and coherency of the network $\left(G_{\mathbf{2}}\right)$.

Macro-criterion $G_{\mathbf{1}}$ aggregates the social subcriterion $\left(G_{(\mathbf{1}, 1)}\right)$ and the economic subcriterion $\left(G_{(\mathbf{1}, 2)}\right)$, while Macro-criterion $G_{\mathbf{2}}$ aggregates the functionality of the network subcriterion $\left(G_{(\mathbf{2}, 1)}\right)$ and the coherency of the network subcriterion $\left(G_{(\mathbf{2}, 2)}\right)$. Under these four subcriteria, 11 elementary criteria are considered: 
- Social $\left(G_{(\mathbf{1}, 1)}\right)$ :

- level of the transport demand $\left(g_{(\mathbf{1}, 1,1)}\right)$,

- degree of urgency $\left(g_{(\mathbf{1}, 1,2)}\right)$,

- environmental effects $\left(g_{(\mathbf{1}, 1,3)}\right)$;

- Economic $\left(G_{(\mathbf{1}, 2)}\right)$ :

- cost effectiveness $\left(g_{(\mathbf{1}, 2,1)}\right)$,

- relative investment cost $\left(g_{(\mathbf{1}, 2,2)}\right)$,

- financing feasibility $\left(g_{(\mathbf{1}, 2,3)}\right)$;

- Functionality of the network $\left(G_{(\mathbf{2}, 1)}\right)$ :

- international importance of the link $\left(g_{(\mathbf{2}, 1,1)}\right)$,

- share of international transport in the total transport demand for passengers $\left(g_{(\mathbf{2}, 1,2)}\right)$,

- share of international transport in the total transport demand for goods $\left(g_{(\mathbf{2}, 1,3)}\right)$;

- Coherency of the network $\left(G_{(\mathbf{2}, 2)}\right)$ :

- interconnection of existing networks $\left(g_{(\mathbf{2}, 2,1)}\right)$;

- meeting EU standards of service $\left(g_{(\mathbf{2}, 2,2)}\right)$.

Figure 3: Hierarchy of Criteria

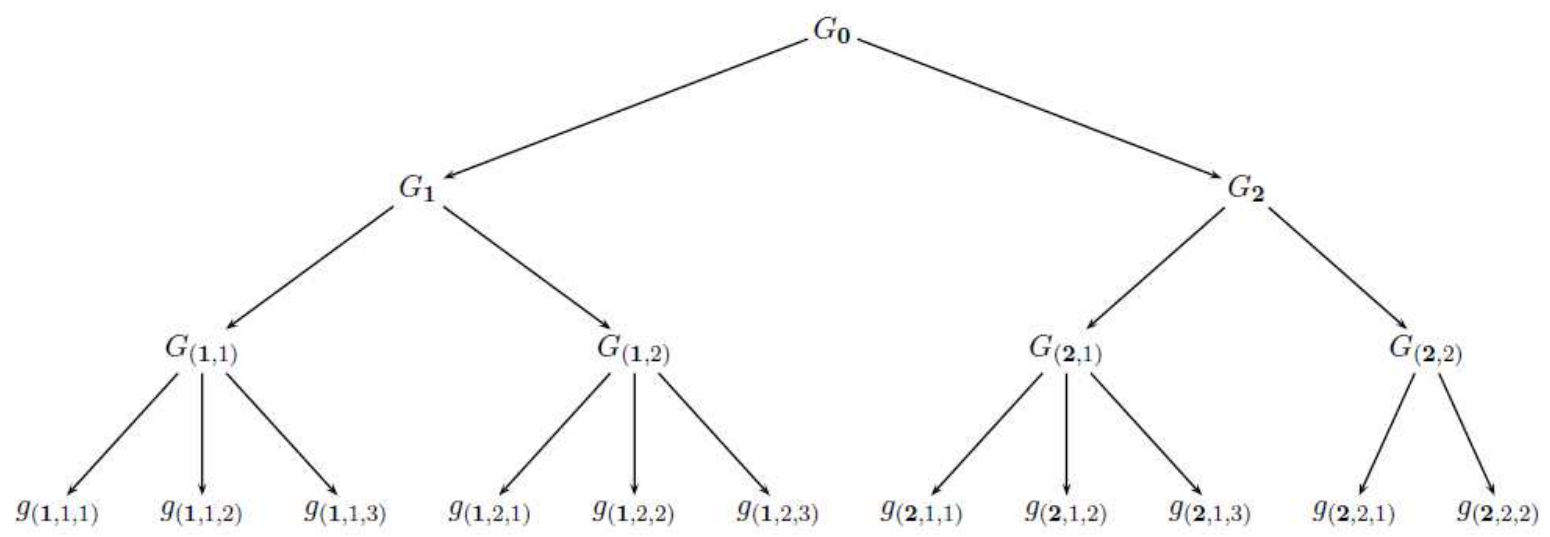

The scores given to the projects by the team of experts on the 11 elementary criteria range from $\mathrm{F}$ (the worst) to A (the best). To simplify the definition of reference profiles and comparison thresholds, the qualitative scale F-A was converted to the interval scale 0-10. In Table 3, we provide performances of some of the 223 projects. 
Table 3: Performances of projects on 11 elementary criteria

\begin{tabular}{|c|ccc|ccc|ccc|cc|}
\hline Project & $g_{(\mathbf{1}, \mathbf{1}, \mathbf{1})}$ & $g_{(\mathbf{1}, \mathbf{1}, \mathbf{2})}$ & $g_{(\mathbf{1}, \mathbf{1}, \mathbf{3})}$ & $g_{(\mathbf{1}, \mathbf{2}, \mathbf{1})}$ & $g_{(\mathbf{1}, \mathbf{2}, \mathbf{2})}$ & $g_{(\mathbf{1}, \mathbf{2}, \mathbf{3})}$ & $g_{(\mathbf{2}, \mathbf{1}, \mathbf{1})}$ & $g_{(\mathbf{2}, \mathbf{1}, \mathbf{2})}$ & $g_{(\mathbf{2}, \mathbf{1}, \mathbf{3})}$ & $g_{(\mathbf{2}, \mathbf{2}, \mathbf{1})}$ & $g_{(\mathbf{2}, \mathbf{2}, \mathbf{2})}$ \\
\hline$a_{13}$ & 4 & 2 & 4 & 0 & 6 & 4 & 8 & 2 & 2 & 6 & 8 \\
$a_{93}$ & 8 & 10 & 2 & 4 & 2 & 6 & 8 & 8 & 6 & 10 & 4 \\
$\ldots$ & $\ldots$ & $\ldots$ & $\ldots$ & $\ldots$ & $\ldots$ & $\ldots$ & $\ldots$ & $\ldots$ & $\ldots$ & $\ldots$ & $\ldots$ \\
$\ldots$ & $\ldots$ & $\ldots$ & $\ldots$ & $\ldots$ & $\ldots$ & $\ldots$ & $\ldots$ & $\ldots$ & $\ldots$ & $\ldots$ & $\ldots$ \\
$\ldots$ & $\ldots$ & $\ldots$ & $\ldots$ & $\ldots$ & $\ldots$ & $\ldots$ & $\ldots$ & $\ldots$ & $\ldots$ & $\ldots$ & $\ldots$ \\
$a_{211}$ & 8 & 2 & 0 & 2 & 4 & 4 & 8 & 2 & 8 & 6 & 6 \\
\hline
\end{tabular}

All elementary criteria have weight $w_{\mathbf{t}}=0.1$, except elementary criteria $g_{(\mathbf{2}, 1,2)}$ and $g_{(\mathbf{2}, 1,3)}$ having weight $w_{\mathbf{t}}=0.05$.

Comparing to the original TIRS study, we shall consider only three, and not four categories, to which each project can be assigned with respect to its feasibility of realization. We shall denote them by $C l_{1}, C l_{2}$ and $\mathrm{Cl}_{3}$, and they will contain, respectively, projects to be discarded, projects requiring further investigation for a final decision, and projects which may be funded and implemented rapidly.

In Table 4, we show the performances of reference profiles on the 11 elementary criteria and the corresponding indifference, preference and veto thresholds. Having three categories, we need to define only two profiles, and we suppose that they have the same indifference, preference and veto thresholds on all elementary criteria.

Table 4: Reference profiles defined on the 11 elementary criteria and the corresponding thresholds

\begin{tabular}{|c|ccc|ccc|ccc|cc|}
\hline Profile & $g_{(\mathbf{1}, \mathbf{1}, \mathbf{1})}$ & $g_{(\mathbf{1}, \mathbf{1}, \mathbf{2})}$ & $g_{(\mathbf{1}, \mathbf{1}, \mathbf{3})}$ & $g_{(\mathbf{1 , 2}, \mathbf{1})}$ & $g_{(\mathbf{1}, \mathbf{2}, \mathbf{2})}$ & $g_{(\mathbf{1}, \mathbf{2}, \mathbf{3})}$ & $g_{(\mathbf{2}, \mathbf{1}, \mathbf{1})}$ & $g_{(\mathbf{2}, \mathbf{1}, \mathbf{2})}$ & $g_{(\mathbf{2}, \mathbf{1}, \mathbf{3})}$ & $g_{(\mathbf{2}, \mathbf{2}, \mathbf{1})}$ & $g_{(\mathbf{2}, \mathbf{2}, \mathbf{2})}$ \\
\hline$b_{1}$ & 3 & 4 & 2.5 & 3 & 2.5 & 3 & 3 & 2.5 & 2.5 & 3.5 & 3.5 \\
$b_{2}$ & 7 & 8 & 6.5 & 7 & 6.5 & 7 & 7 & 6.5 & 6.5 & 7 & 7.5 \\
\hline$q_{\mathbf{t}}$ & 1 & 1 & 1 & 1 & 1 & 1 & 1 & 1 & 1 & 1 & 1 \\
$p_{\mathbf{t}}$ & 2 & 2 & 2 & 2 & 2 & 2 & 2 & 2 & 2 & 2 & 2 \\
$v_{\mathbf{t}}$ & 4 & 4 & 4 & 4 & 4 & 4 & 4 & 4 & 4 & 4 & 4 \\
\hline
\end{tabular}

We decided that each cutting level $\lambda_{\mathbf{r}}, \mathbf{r} \in L B O$, is equal to $70 \%$ of the sum of weights of elementary criteria descending from $G_{\mathbf{r}}$.

Table 5: Cutting levels

\begin{tabular}{|c|cc|cc|c|c|c|}
\hline Cutting level & $G_{(\mathbf{1}, \mathbf{1})}$ & $G_{(\mathbf{1}, \mathbf{2})}$ & $G_{(\mathbf{2}, \mathbf{1})}$ & $G_{(\mathbf{2}, \mathbf{2})}$ & $G_{(\mathbf{1})}$ & $G_{(\mathbf{2})}$ & $G_{(\mathbf{0})}$ \\
\hline$\lambda_{\mathbf{r}}$ & 0.21 & 0.21 & 0.14 & 0.14 & 0.42 & 0.28 & 0.7 \\
\hline
\end{tabular}

In Table 6, we report the classifications of some projects with respect to all criteria and subcriteria in the hierarchy, considering both, optimistic and pessimistic assignment procedures.

Let us observe that when one is using the optimistic assignment procedure, even if project $a_{211}$ is assigned to category $C l_{2}$ with respect to criteria $G_{(\mathbf{1}, 1)}$ and $G_{(\mathbf{1}, 2)}$, it is assigned to category $C l_{1}$ with respect to criterion $G_{\mathbf{1}}$. As already observed in section 4 and deeply investigated in Appendix B, the reason is that 
Table 6: Classification of projects: at the comprehensive level, and at the first and the second level of the hierarchy tree

(a) Pessimistic assignment

\begin{tabular}{|c|cc|cc|c|c|c|}
\hline Project & $G_{(\mathbf{1}, \mathbf{1})}$ & $G_{(\mathbf{1}, \mathbf{2})}$ & $G_{(\mathbf{2}, \mathbf{1})}$ & $G_{(\mathbf{2}, \mathbf{2})}$ & $G_{(\mathbf{1})}$ & $G_{(\mathbf{2})}$ & $G_{(\mathbf{0})}$ \\
\hline$a_{13}$ & $C l_{1}$ & $C l_{1}$ & $C l_{2}$ & $C l_{3}$ & $C l_{1}$ & $\mathrm{Cl}_{2}$ & $C l_{2}$ \\
$a_{93}$ & $C l_{2}$ & $C l_{2}$ & $C l_{3}$ & $\mathrm{Cl}_{2}$ & $\mathrm{Cl}_{2}$ & $\mathrm{Cl}_{3}$ & $\mathrm{Cl}_{2}$ \\
$a_{211}$ & $\mathrm{Cl}_{1}$ & $\mathrm{Cl}_{2}$ & $\mathrm{Cl}_{3}$ & $\mathrm{Cl}_{2}$ & $\mathrm{Cl}_{1}$ & $\mathrm{Cl}_{2}$ & $\mathrm{Cl}_{2}$ \\
\hline
\end{tabular}

(b) Optimistic assignment

\begin{tabular}{|c|cc|cc|c|c|c|}
\hline Project & $G_{(\mathbf{1}, \mathbf{1})}$ & $G_{(\mathbf{1}, \mathbf{2})}$ & $G_{(\mathbf{2}, \mathbf{1})}$ & $G_{(\mathbf{2}, \mathbf{2})}$ & $G_{(\mathbf{1})}$ & $G_{(\mathbf{2})}$ & $G_{(\mathbf{0})}$ \\
\hline$a_{13}$ & $\mathrm{Cl}_{1}$ & $\mathrm{Cl}_{2}$ & $C l_{2}$ & $\mathrm{Cl}_{3}$ & $\mathrm{Cl}_{1}$ & $\mathrm{Cl}_{2}$ & $\mathrm{Cl}_{2}$ \\
$a_{93}$ & $\mathrm{Cl}_{3}$ & $\mathrm{Cl}_{2}$ & $\mathrm{Cl}_{3}$ & $\mathrm{Cl}_{3}$ & $\mathrm{Cl}_{2}$ & $\mathrm{Cl}_{3}$ & $\mathrm{Cl}_{2}$ \\
$\mathrm{a}_{211}$ & $\mathrm{Cl}_{2}$ & $\mathrm{Cl}_{2}$ & $\mathrm{Cl}_{3}$ & $\mathrm{Cl}_{2}$ & $\mathrm{Cl}_{1}$ & $\mathrm{Cl}_{2}$ & $\mathrm{Cl}_{2}$ \\
\hline
\end{tabular}

profile $b_{1}$ is not preferred to $a_{211}$, neither on criterion $G_{(\mathbf{1}, 1)}$ nor on criterion $G_{(\mathbf{1}, 2)}$, however, it is preferred to $a_{211}$ on criterion $G_{\mathbf{1}}$.

\section{Conclusions}

In this paper, we presented an extension of the very well known family of ELECTRE Tri sorting methods, ELECTRE Tri-B [21], ELECTRE Tri-C [2] and ELECTRE Tri-nC [3], to decision problems with multiple criteria structured in a hierarchical way. To this end, we applied the Multiple Criteria Hierarchy Process (MCHP) proposed recently in [10]. Application of MCHP permits to get recommendations on the assignments of alternatives not only at the comprehensive level but also in all particular nodes of the hierarchy tree until the last but one level. In particular, regarding the extension of the ELECTRE Tri-B method, we introduced two properties of coherence for outranking with respect to a given criterion, taking into account outranking on criteria immediately descending from it. In this context we singled out some conditions on the cutting levels that proved to be necessary and sufficient to ensure the consistency of the outranking in the hierarchy.

In the paper, we considered only the case where the DM is able to provide a direct preference information, that is, she can give values to all involved parameters (weights of criteria, indifference, preference and veto thresholds, as well as cutting levels). In this context, we showed how to fix the cutting levels so as to respect the coherence properties $\mathbf{C 1}$ ) and C2). We provided a theorem specifying necessary and sufficient conditions for these two properties to be satisfied. It is worth stressing that coherence properties C1) and C2) do not hold for hierarchical versions of ELECTRE Tri sorting methods only, but also for other types of ELECTRE methods applied within the MCHP framework to choice and ranking problems. We took into account the possibility of handling interactions between criteria in a way similar to [13], and we extended the SRF method [14] to compute the weights of criteria when they are organized in a hierarchical structure. The presented extension of the SRF method can be applied to all types of decision problems (choice, ranking and sorting problems) with a hierarchical structure of the set of criteria aggregated in a non-compensatory way. The proposed methodology has been illustrated by a didactic example where we applied the hierarchical ELECTRE Tri-B method to a real world decision problem.

In a future study, we plan to take into account the indirect preference information elicited by the DM in 
terms of some decision examples. To this end, we plan to apply the Robust Ordinal Regression (ROR) $[8,9,15]$ that considers simultaneously all sets of preference model parameters compatible with the preference information provided by the DM. Moreover, other interesting directions of research could be:

- investigation of the relationship between the assignments obtained by the hierarchical ELECTRE TriB and ELECTRE Tri-C methods, similarly to what has been already done in [6] in case of a flat structure of criteria,

- generalization of the SRF method to the case of group decision making and, in particular, to problems with a hierarchical structure of criteria. This could be useful to deal with real world group decision making problems as that one recently considered in [16], where the SRF method has been applied to obtain the weights of criteria for each DM.

\section{Acknowledgments}

The first two authors wish to acknowledge funding by the "Programma Operativo Regionale L.I. 4.1.1.1 PO FESR 2007-2013" within the project "N.U.M.I".

The third author wishes to acknowledge financial support from the Polish National Science Centre, DEC2015/18/A/ST6/00171.

\section{References}

[1] Transport Infrastructure Regional Study (TIRS) in the Balkans. http://www. internationaltransportforum.org/IntOrg/ecmt/southeast/TIRS.html.

[2] J. Almeida Dias, J. Figueira, and B. Roy. ELECTRE TRI-C: A multiple criteria sorting method based on characteristic reference actions. European Journal of Operational Research, 204:565-580, 2010.

[3] J. Almeida Dias, J. Figueira, and B. Roy. A multiple criteria sorting method where each category is characterized by several reference actions: The Electre Tri-nC method. European Journal of Operational Research, 217(3):567-579, 2012.

[4] S. Angilella, S. Corrente, S. Greco, and R. Słowiński. Multiple Criteria Hierarchy Process for the Choquet integral. In R.C. Purshouse et al., editor, EMO 2013, volume 7811 of LNCS, pages 475-489. Springer, Berlin, 2013.

[5] V. Belton and T.J. Stewart. Multiple criteria decision analysis: an integrated approach. Springer, 2002. 
[6] D. Bouyssou and T. Marchant. On the relations between ELECTRE TRI-B and ELECTRE TRI-C and on a new variant of ELECTRE TRI-B. European Journal of Operational Research, 242(1):201-211, 2015.

[7] J.P. Brans and Ph. Vincke. A preference ranking organisation method: The PROMETHEE method for MCDM. Management Science, 31(6):647-656, 1985.

[8] S. Corrente, S. Greco, M. Kadziński, and R. Słowiński. Robust ordinal regression in preference learning and ranking. Machine Learning, 93(2-3):381-422, 2013.

[9] S. Corrente, S. Greco, M. Kadziński, and R. Słowiński. Robust Ordinal Regression. Wiley Encyclopedia of Operations Research and Management Science, pages 1-10, 2014.

[10] S. Corrente, S. Greco, and R. Słowiński. Multiple Criteria Hierarchy Process in Robust Ordinal Regression. Decision Support Systems, 53(3):660-674, 2012.

[11] S. Corrente, S. Greco, and R. Słowiński. Multiple Criteria Hierarchy Process with ELECTRE and PROMETHEE. Omega, 41:820-846, 2013.

[12] J. Figueira, S. Greco, and M. Ehrgott (eds.). Multiple Criteria Decision Analysis: State of the Art Surveys. Springer, Berlin, 2005.

[13] J. Figueira, S. Greco, and B. Roy. ELECTRE methods with interaction between criteria: An extension of the concordance index. European Journal of Operational Research, 199(2):478-495, 2009.

[14] J. Figueira and B. Roy. Determining the weights of criteria in the ELECTRE type methods with a revised Simos' procedure. European Journal of Operational Research, 139:317-326, 2002.

[15] S. Greco, V. Mousseau, and R. Słowiński. Ordinal regression revisited: multiple criteria ranking using a set of additive value functions. European Journal of Operational Research, 191(2):416-436, 2008.

[16] D.C. Morais, A.T. de Almeida, and J.R. Figueira. A sorting model for group decision making: A case study of water losses in Brazil. Group Decision and Negotiation, 23(5):937-960, 2014.

[17] J. Moscarola and B. Roy. Procédure automatique d'examen de dossiers fondée sur une segmentation trichotomique en présence de critères multiples. RAIRO-Operations Research, 11(2):145-173, 1977.

[18] P. Nemery and C. Lamboray. FlowSort: a flow-based sorting method with limiting or central profiles. TOP, 16:90-113, 2008. 
[19] J. Simos. Evaluer l'impact sur l'environnement: Une approche originale par l'analyse multicritère et la négociation. Presses Polytechniques et Universitaires Romandes, Lausanne, 1990.

[20] J. Simos. L'evaluation environnementale: Un processus cognitif négocié. Ph.D. Thesis. DGF-EPFL, Lausanne, 1990.

[21] W. Yu. Aide multicritère à la décision dans le cadre de la problématique du tri: méthodes et applications. Ph.D. thesis. LAMSADE, Université Paris Dauphine, Paris, 1992.

\section{Appendix A}

\section{Proof of Lemma 4.1}

1. Since $a$ dominates $b$ with respect to criterion $G_{\mathbf{r}}$, for each $\mathbf{t} \in E\left(G_{\mathbf{r}}\right)$ we have $g_{\mathbf{t}}(c)-g_{\mathbf{t}}(a) \leq g_{\mathbf{t}}(c)-g_{\mathbf{t}}(b)$. Because $\phi_{\mathbf{t}}(x, y)$ is a non-increasing function of $g_{\mathbf{t}}(y)-g_{\mathbf{t}}(x)$, then $\phi_{\mathbf{t}}(a, c) \geq \phi_{\mathbf{t}}(b, c)$ for all $\mathbf{t} \in E\left(G_{\mathbf{r}}\right)$. From this, it follows that

$$
C_{\mathbf{r}}(a, c) \geq C_{\mathbf{r}}(b, c)
$$

- When outranking $\mathbf{O 1})$ is considered, since $b S_{\mathbf{r}} c$ is equivalent to $C_{\mathbf{r}}(b, c) \geq \lambda_{\mathbf{r}}$, and taking into account equation (14), it follows that $C_{\mathbf{r}}(a, c) \geq \lambda_{\mathbf{r}}$, which proves the thesis;

- When outranking O2) is considered, $b S_{\mathbf{r}} c$ implies that $g_{\mathbf{t}}(c)-g_{\mathbf{t}}(b)<v_{\mathbf{t}}$ for all $\mathbf{t} \in E\left(G_{\mathbf{r}}\right)$, and, therefore, $g_{\mathbf{t}}(c)-g_{\mathbf{t}}(a)<v_{\mathbf{t}}$ for all $\mathbf{t} \in E\left(G_{\mathbf{r}}\right)$ implying, together with the point proved above, the thesis;

- When outranking O3) is considered, two things should be observed:

- since $d_{\mathbf{t}}(x, y)$ is a non-decreasing function of the difference $g_{\mathbf{t}}(y)-g_{\mathbf{t}}(x)$, we have for all $\mathbf{t} \in E\left(G_{\mathbf{r}}\right)$ that $d_{\mathbf{t}}(a, c) \leq d_{\mathbf{t}}(b, c)$;

$$
-\bar{F}_{\mathbf{r}}(a, c)=\left\{\mathbf{t} \in E\left(G_{\mathbf{r}}\right): d_{\mathbf{t}}(a, c)>C_{\mathbf{r}}(a, c)\right\} \subseteq \bar{F}_{\mathbf{r}}(b, c)=\left\{\mathbf{t} \in E\left(G_{\mathbf{r}}\right): d_{\mathbf{t}}(b, c)>C_{\mathbf{r}}(b, c)\right\} .
$$

From the previous two points, and taking into account that $\frac{1-d_{\mathbf{t}}(b, c)}{1-C_{\mathbf{r}}(b, c)}<1$ when $\mathbf{t} \in \bar{F}_{\mathbf{r}}(b, c)$ $\left(d_{\mathbf{t}}(a, b)\right.$ and $C_{\mathbf{r}}(a, b)$ being non negative values), by equation (14) it follows that

$$
\sigma_{\mathbf{r}}(a, c)=C_{\mathbf{r}}(a, c) \prod_{\mathbf{t} \in \bar{F}_{\mathbf{r}}(a, c)} \frac{1-d_{\mathbf{t}}(a, c)}{1-C_{\mathbf{r}}(a, c)} \geq C_{\mathbf{r}}(b, c) \prod_{\mathbf{t} \in \bar{F}_{\mathbf{r}}(b, c)} \frac{1-d_{\mathbf{t}}(b, c)}{1-C_{\mathbf{r}}(b, c)}=\sigma_{\mathbf{r}}(b, c) .
$$

Since $b S_{\mathbf{r}} c$ is equivalent to $\sigma_{\mathbf{r}}(b, c) \geq \lambda_{\mathbf{r}}$, from equation (15) it follows that $\sigma_{\mathbf{r}}(a, c) \geq \lambda_{\mathbf{r}}$, which proves the thesis.

2. Points 2,3 , and 4 , are proved analogously. 


\section{Proof of Theorem 4.1}

For the sake of simplicity and without loss of generality, we shall suppose that criterion $G_{\mathbf{r}}, \mathbf{r} \in \mathcal{I}_{\mathcal{G}} \backslash E L$, has only two subcriteria $G_{(\mathbf{r}, 1)}$ and $G_{(\mathbf{r}, 2)}$, and $a \underset{(\mathbf{r}, 1)}{\longrightarrow} C l_{k_{1}}, a \underset{(\mathbf{r}, 2)}{\longrightarrow} C l_{k_{2}}$. Let us suppose that $k_{1}<k_{2}$; as a consequence, $k_{1}=\min \left\{k_{1}, k_{2}\right\}$ and $k_{2}=\max \left\{k_{1}, k_{2}\right\}$.

1. Considering the pessimistic assignment,

(a) $a \underset{(\mathbf{r}, 1)}{\longrightarrow} C l_{k_{1}}$ means that $\operatorname{not}\left(a S_{(\mathbf{r}, 1)} b_{h}\right), h=p, \ldots, k_{1}$, and $a S_{(\mathbf{r}, 1)} b_{k_{1}-1}$,

(b) $a \underset{(\mathbf{r}, 2)}{\longrightarrow} C l_{k_{2}}$ means that $\operatorname{not}\left(a S_{(\mathbf{r}, 2)} b_{h}\right), h=p, \ldots, k_{2}$, and $a S_{(\mathbf{r}, 2)} b_{k_{2}-1}$.

From (a) and (b), it follows that $\operatorname{not}\left(a S_{(\mathbf{r}, 1)} b_{h}\right)$ and $\operatorname{not}\left(a S_{(\mathbf{r}, 2)} b_{h}\right)$ for all $h=k_{2}, \ldots, p$. Therefore, by condition C2) we get that $\operatorname{not}\left(a S_{\mathbf{r}} b_{h}\right)$ for all $h=k_{2}, \ldots, p$. As a consequence, $a \underset{\mathbf{r}}{\rightarrow} C l_{k}$ with $k \leq k_{2}$. Analogously, since $b_{k_{2}-1}$ dominates $b_{k_{1}-1}$ and $a S_{(\mathbf{r}, 2)} b_{k_{2}-1}$, by point 4.1 of Lemma 4.1 , we have that $a S_{(\mathbf{r}, 2)} b_{k_{1}-1}$. As a consequence, by condition C1) we get that $a S_{\mathbf{r}} b_{k_{1}-1}$ and therefore $a \underset{\mathbf{r}}{\rightarrow} C l_{k}$ with $k \geq k_{1}$.

2. Considering the optimistic assignment,

(a) $a \underset{(\mathbf{r}, 1)}{\longrightarrow} C l_{k_{1}}$ means that $\operatorname{not}\left(b_{h} \succ_{(\mathbf{r}, 1)} a\right), h=1, \ldots, k_{1}-1$, and $b_{k_{1}} \succ_{(\mathbf{r}, 1)} a$,

(b) $a \underset{(\mathbf{r}, 2)}{\longrightarrow} C l_{k_{2}}$ means that $\operatorname{not}\left(b_{h} \succ_{(\mathbf{r}, 2)} a\right), h=1, \ldots, k_{2}-1$, and $b_{k_{2}} \succ_{(\mathbf{r}, 2)} a$.

Before proceeding with the proof of this point, by coherence properties C1) and C2) it is straightforward proving that, if $a \succ_{(\mathbf{r}, j)} b$ for all $j=1, \ldots, n(\mathbf{r})$, then $a \succ_{\mathbf{r}} b$, and by Lemma 4.1, if $b_{h} \succ_{\mathbf{r}} a$, then $b_{l} \succ_{\mathbf{r}} a$ for all $l>h$.

Since, for the hypothesis, $b_{k_{1}} \succ_{(\mathbf{r}, 1)} a$, and $k_{2}>k_{1}$, we have that $b_{k_{2}} \succ_{(\mathbf{r}, 1)} a$. Consequently, $b_{k_{2}} \succ_{(\mathbf{r})} a$. This means that $a \underset{\mathbf{r}}{\rightarrow} C l_{k}$ with $k \leq k_{2}$.

\section{Appendix B}

The unexpected result for which an alternative $a$ can be assigned on criterion $G_{\mathbf{r}}$ to a category $C l_{k}$ with $k<\min \left\{k_{1}, \ldots, k_{n(\mathbf{r})}\right\}$, where $C l_{k_{1}}, \ldots, C l_{k_{n(\mathbf{r})}}$ are the categories to which $a$ is assigned on subcriteria 
$G_{(\mathbf{r}, 1)}, \ldots, G_{(\mathbf{r}, n(\mathbf{r}))}$, is due to the following statement:

$$
\operatorname{not}\left(a \succ_{(\mathbf{r}, j)} b\right) \text {, for all } j=1, \ldots, n(\mathbf{r}) \text { “does not imply" } \operatorname{not}\left(a \succ_{\mathbf{r}} b\right) \text {. }
$$

Indeed, as we shall show in the following, in general, the fact that $a$ is not preferred to $b$ on all subcriteria $G_{(\mathbf{r}, j)}$ of criterion $G_{\mathbf{r}}$ implies that $a$ is not preferred to $b$ on criterion $G_{\mathbf{r}}$. Only in two cases, nothing could be said about the preference of $a$ over $b$ on criterion $G_{\mathbf{r}}$. Let us analyze in which cases it could happen.

Remember that $\operatorname{not}\left(a \succ_{(\mathbf{r}, j)} b\right)$ for all $j=1, \ldots, n(\mathbf{r})$. This means that for all $j=1, \ldots, n(\mathbf{r})$

$$
\operatorname{not}\left[a S_{(\mathbf{r}, j)} b \wedge \operatorname{not}\left(b S_{(\mathbf{r}, j)} a\right)\right] \equiv\left[\operatorname{not}\left(a S_{(\mathbf{r}, j)} b\right) \vee b S_{(\mathbf{r}, j)} a\right]
$$

Consequently, for all $j=1, \ldots, n(\mathbf{r})$, the fact that $\left[\operatorname{not}\left(a S_{(\mathbf{r}, j)} b\right) \vee b S_{(\mathbf{r}, j)} a\right]$ can be true in three cases:

- $\operatorname{not}\left(a S_{(\mathbf{r}, j)} b\right)$ and $b S_{(\mathbf{r}, j)} a \quad\left(b \succ_{(\mathbf{r}, j)} a\right)$

- $\operatorname{not}\left(a S_{(\mathbf{r}, j)} b\right)$ and $\operatorname{not}\left(b S_{(\mathbf{r}, j)} a\right) \quad\left(a ?_{(\mathbf{r}, j)} b\right)$,

- $a S_{(\mathbf{r}, j)} b$ and $b S_{(\mathbf{r}, j)} a \quad\left(b \sim_{(\mathbf{r}, j)} a\right)$.

Now, let define the sets $J_{\mathbf{r}}^{R}, J_{\mathbf{r}}^{P^{-1}}, J_{\mathbf{r}}^{I} \subseteq\{1, \ldots, n(\mathbf{r})\}$, such that:

1. $j \in J_{\mathbf{r}}^{P^{-1}}$ iff $b \succ_{(\mathbf{r}, j)} a$,

2. $j \in J_{\mathbf{r}}^{R}$ iff $a ?{ }_{(\mathbf{r}, j)} b$,

3. $j \in J_{\mathbf{r}}^{I}$ iff $a \sim(\mathbf{r}, j) b$.

We can observe the following cases:

$\left.\gamma_{1}\right)$ If $J_{\mathbf{r}}^{R}=\{1, \ldots, n(\mathbf{r})\}$ (that is $a$ is incomparable to $b$ on all subcriteria $G_{(\mathbf{r}, j)}$ of criterion $G_{\mathbf{r}}$ ), then $a ? \mathbf{r} b$ and, consequently, $\operatorname{not}\left(a \succ_{\mathbf{r}} b\right)$;

Indeed, for all $j=1, \ldots, n(\mathbf{r}), a ?_{(\mathbf{r}, j)} b$ implies that $\operatorname{not}\left(a S_{(\mathbf{r}, j)} b\right)$ and $\operatorname{not}\left(b S_{(\mathbf{r}, j)} a\right)$ for all $j=1, \ldots, n(\mathbf{r})$.

By the coherence property $\mathbf{C 2})$ we have that $\operatorname{not}\left(a S_{\mathbf{r}} b\right)$ and $\operatorname{not}\left(b S_{\mathbf{r}} a\right)$ and, therefore, $a ? \mathbf{r} b$.

$\gamma_{2}$ ) If $J_{\mathbf{r}}^{P^{-1}}=\{1, \ldots, n(\mathbf{r})\}$ (that is $b$ is preferred to $a$ on all subcriteria $G_{(\mathbf{r}, j)}$ of criterion $G_{\mathbf{r}}$ ), then $b \succ_{\mathbf{r}} a$ and, consequently, $\operatorname{not}\left(a \succ_{\mathbf{r}} b\right)$;

Indeed, for all $j=1, \ldots, n(\mathbf{r}), b \succ_{(\mathbf{r}, j)} a$ implies that $\operatorname{not}\left(a S_{(\mathbf{r}, j)} b\right)$ and $b S_{(\mathbf{r}, j)} a$ for all $j=1, \ldots, n(\mathbf{r})$. By the coherence properties C2) and C1) we have that $\operatorname{not}\left(a S_{\mathbf{r}} b\right)$ and $b S_{\mathbf{r}} a$. Therefore $b \succ_{\mathbf{r}} a$.

$\left.\gamma_{3}\right)$ If $J_{\mathbf{r}}^{I}=\{1, \ldots, n(\mathbf{r})\}$ (that is $a$ is indifferent to $b$ on all subcriteria $G_{(\mathbf{r}, j)}$ of criterion $G_{\mathbf{r}}$ ), then $a \sim_{\mathbf{r}} b$ and, consequently, $\operatorname{not}\left(a \succ_{\mathbf{r}} b\right)$;

Indeed, for all $j=1, \ldots, n(\mathbf{r}), a \sim_{(\mathbf{r}, j)} b$ implies that $a S_{(\mathbf{r}, j)} b$ and $b S_{(\mathbf{r}, j)} a$ for all $j=1, \ldots, n(\mathbf{r})$. By the coherence property $\mathbf{C 1}$ ) we have that $a S_{\mathbf{r}} b$ and $b S_{\mathbf{r}} a$. Therefore $a \sim_{\mathbf{r}} b$. 
$\left.\gamma_{4}\right)$ If $J_{\mathbf{r}}^{I}=\emptyset, J_{\mathbf{r}}^{R} \neq \emptyset$ and $J_{\mathbf{r}}^{P^{-1}} \neq \emptyset$ then $\operatorname{not}\left(a \succ_{\mathbf{r}} b\right)$. Indeed, the hypothesis implies that $a$ ? $\mathbf{r}_{\mathbf{r}, j)} b$ for $j \in J_{\mathbf{r}}^{R}$ and $b \succ_{(\mathbf{r}, j)} a$ for $j \in J_{\mathbf{r}}^{P^{-1}}$ while $a$ is not indifferent to $b$ for any $j \in\{1, \ldots, n(\mathbf{r})\}$. By the hypothesis, we have that $\operatorname{not}\left(a S_{(\mathbf{r}, j)} b\right)$ for all $j=1, \ldots, n(\mathbf{r})$ that, by the coherence property $\left.\mathbf{C 2}\right)$ implies $\operatorname{not}\left(a S_{\mathbf{r}} b\right)$ and, therefore, $\operatorname{not}\left(a \succ_{\mathbf{r}} b\right)$.

$\left.\gamma_{5}\right)$ If $J_{\mathbf{r}}^{R}=\emptyset, J_{\mathbf{r}}^{I} \neq \emptyset$ and $J_{\mathbf{r}}^{P^{-1}} \neq \emptyset$ then $\operatorname{not}\left(a \succ_{\mathbf{r}} b\right)$. Indeed, the hypothesis implies that $a \sim_{(\mathbf{r}, j)} b$ for $j \in J_{\mathbf{r}}^{I}$ and $b \succ_{(\mathbf{r}, j)} a$ for $j \in J_{\mathbf{r}}^{P^{-1}}$ while $a$ is not incomparable with $b$ for any $j \in\{1, \ldots, n(\mathbf{r})\}$. By the hypothesis, we have that $b S_{(\mathbf{r}, j)} a$ for all $j=1, \ldots, n(\mathbf{r})$ that, by the coherence property $\mathbf{C 1}$ ) implies $b S_{\mathbf{r}} a$ and, therefore, $\operatorname{not}\left(a \succ_{\mathbf{r}} b\right)$.

$\left.\gamma_{6}\right)$ If $J_{\mathbf{r}}^{R} \neq \emptyset, J_{\mathbf{r}}^{I} \neq \emptyset$ and $J_{\mathbf{r}}^{P^{-1}}=\emptyset$ then nothing could be said about the preference of $a$ over $b$ on criterion $G_{\mathbf{r}}$. It is still possible that $a \succ_{\mathbf{r}} b$ or that $\operatorname{not}\left(a \succ_{\mathbf{r}} b\right)$.

$\gamma_{7}$ ) If $J_{\mathbf{r}}^{R} \neq \emptyset, J_{\mathbf{r}}^{I} \neq \emptyset$ and $J_{\mathbf{r}}^{P^{-1}} \neq \emptyset$ then nothing could be said about the preference of $a$ over $b$ on criterion $G_{\mathbf{r}}$. It is still possible that $a \succ_{\mathbf{r}} b$ or that $\operatorname{not}\left(a \succ_{\mathbf{r}} b\right)$.

As a consequence of this analysis, we underline that two cases could be "troubling", that are cases $\gamma_{6}$ ) and $\gamma_{7}$ ) and thus, the hypotheses of cases $\gamma_{6}$ ) and $\gamma_{7}$ ) are not in contradiction with the fact that $a \succ_{\mathbf{r}} b$. Indeed, in one case, if $a$ is incomparable with $b$ for some subcriteria $G_{(\mathbf{r}, j)}$ and $a$ is indifferent to $b$ for some subcriteria $G_{(\mathbf{r}, j)}$, it is still possible that $a$ is preferred to $b$ on criterion $G_{\mathbf{r}}$. Analogously, even if $a$ is indifferent to $b$ for some subcriteria, incomparable for others, and there is a strict preference of $b$ over $a$ on some others, it is still possible that $a$ is preferred to $b$ on criterion $G_{\mathbf{r}}$. 